\title{
On the Facets of Mixed Integer Programs with Two Integer Variables and Two Constraints
}

\author{
Gérard Cornuéjols * \\ Tepper School of Business, Carnegie Mellon University, Pittsburgh, PA 15213 \\ and LIF, Faculté des Sciences de Luminy, Université de Marseille, France \\ gc0v@andrew.cmu.edu \\ and \\ François Margot ${ }^{\dagger}$ \\ Tepper School of Business, Carnegie Mellon University, Pittsburgh, PA 15213 \\ fmargot@andrew.cmu.edu
}

August 2007; revised March 2008

\begin{abstract}
In this paper we consider an infinite relaxation of the mixed integer linear program with two integer variables, nonnegative continuous variables and two equality constraints, and we give a complete characterization of its facets. We also derive an analogous characterization of the facets of the underlying finite integer program.
\end{abstract}

\section{Introduction}

We consider the mixed 2-integer-variable linear program with two constraints

$$
\begin{aligned}
& x=f+\sum_{j=1}^{k} r^{j} s_{j} \\
& x \in \mathbb{Z}^{2} \\
& s \in \mathbb{R}_{+}^{k}
\end{aligned}
$$

where $f \in \mathbb{Q}^{2} \backslash \mathbb{Z}^{2}, k \geq 1$, and $r^{j} \in \mathbb{Q}^{2} \backslash\{0\}$. Let $R_{f}\left(r^{1}, \ldots, r^{k}\right)$ be the convex hull of all vectors $s \in \mathbb{R}_{+}^{k}$ such that $f+\sum_{j=1}^{k} r^{j} s_{j}$ is integral. $R_{f}\left(r^{1}, \ldots, r^{k}\right)$ is a polyhedron (We refer the reader to [17] for standard definitions). Model (1) was considered by Andersen, Louveaux, Weismantel and Wolsey [1]. They showed that the nontrivial facets of $R_{f}\left(r^{1}, \ldots, r^{k}\right)$ are necessarily defined by split inequalities or intersection cuts (Balas [2]) arising from triangles or quadrilaterals in $\mathbb{R}^{2}$. A goal of this paper is to give a converse to the result in [1]: which splits, triangles and quadrilaterals actually define facets of $R_{f}\left(r^{1}, \ldots, r^{k}\right)$ ? We present our analysis in the more general context of the Gomory-Johnson infinite group relaxation.

\footnotetext{
* Supported by NSF grant CMMI0653419, ONR grant N00014-97-1-0196 and ANR grant BLAN06-1-138894.

${ }^{\dagger}$ Supported by ONR grant N00014-97-1-0196.
} 
Gomory [9], Gomory and Johnson [12] and Johnson [13] suggested relaxing the $k$-dimensional space of variables $s=\left(s_{1}, \ldots, s_{k}\right)$ to an infinite-dimensional space, where the variables $s_{r}$ are defined for any $r \in \mathbb{Q}^{2}$. We get the infinite program with two integer variables and two constraints

$$
\begin{aligned}
& x=f+\sum r s_{r} \\
& x \in \mathbb{Z}^{2} \\
& s \geq 0 \text { with finite support. }
\end{aligned}
$$

The vector $s=\left(s_{r}\right)_{r \in \mathbb{Q}^{2}}$ is said to have finite support if $s_{r} \neq 0$ for a finite number of $r \in \mathbb{Q}^{2}$. Let $R_{f}$ be the convex hull of all vectors $s \geq 0$ with finite support such that $f+\sum r s_{r}$ is integral. Note that the polyhedron $R_{f}\left(r^{1}, \ldots, r^{k}\right)$ is obtained from $R_{f}$ by setting $s_{r}=0$ for all $r \in \mathbb{Q}^{2} \backslash\left\{r^{1}, \ldots, r^{k}\right\}$. Our motivation for working with $R_{f}$ is that it only has one parameter, namely $f$, and therefore the results are cleaner than with $R_{f}\left(r^{1}, \ldots, r^{k}\right)$. A drawback of $R_{f}$ is that it is not a closed set. For example, the sequence $s^{k}$ for $k=1,2, \ldots$ defined by

$$
s_{r}^{k}= \begin{cases}\frac{1}{k} & \text { if } r=-k f \\ 0 & \text { otherwise }\end{cases}
$$

is in $R_{f}$ and converges to 0 , but $0 \notin R_{f}$ since $f \in \mathbb{Q}^{2} \backslash \mathbb{Z}^{2}$. Throughout the paper, we relate results obtained for the convex set $R_{f}$ to those obtained for the polyhedron $R_{f}\left(r^{1}, \ldots, r^{k}\right)$.

\subsection{Minimal valid inequalities}

We say that an inequality $\alpha s \geq \beta$ is valid for $R_{f}$ (resp. $R_{f}\left(r^{1}, \ldots, r^{k}\right)$ ) if it is satisfied by all vectors in $R_{f}$ (resp. $R_{f}\left(r^{1}, \ldots, r^{k}\right)$ ). Inequalities $s_{i} \geq 0$ are called trivial valid inequalities. In this paper, we discuss only nontrivial valid inequalities. The solution $s=0$ is not feasible for $R_{f}$. Any valid inequality for $R_{f}$ that cuts off the vector $s=0$ is of the form

$$
\sum \psi(r) s_{r} \geq 1
$$

where $\psi: \mathbb{Q}^{2} \rightarrow \mathbb{R} \cup\{+\infty\}$ and, as above, we only consider vectors $s$ with finite support. To avoid ambiguity, the product $+\infty \cdot 0$ is defined to be 0 .

Any valid inequality for $R_{f}$ yields a valid inequality for $R_{f}\left(r^{1}, \ldots, r^{k}\right)$ by simply restricting it to the space $r^{1}, \ldots, r^{k}$. Furthermore, a full description of the polyhedron $R_{f}\left(r^{1}, \ldots, r^{k}\right)$ is obtained from the set of valid inequalities for $R_{f}$ by adding the constraints $s_{r}=0$ for $r \neq r^{1}, \ldots, r^{k}$. Therefore we will assume in the remainder that valid inequalities for $R_{f}\left(r^{1}, \ldots, r^{k}\right)$ are restrictions of valid inequalities for $R_{f}$.

An inequality $\sum \psi(r) s_{r} \geq 1$ valid for $R_{f}$ is minimal if there is no valid inequality $\sum \psi^{\prime}(r) s_{r} \geq 1$ where $\psi^{\prime} \leq \psi$ and $\psi^{\prime}(r)<\psi(r)$ for at least one $r \in \mathbb{Q}^{2}$. Note that when $\psi(r)=+\infty$ we have $\psi^{\prime}(r)<\psi(r)$ if and only if $\psi^{\prime}(r)$ is finite. If $\psi$ defines a minimal valid inequality $\sum \psi(r) s_{r} \geq 1$, we also say that the function $\psi$ is minimal. In [4], it was shown that a minimal valid function $\psi$ is nonnegative, positively homogeneous, piecewise linear, and convex. Recall that a function $\psi$ is positively homogeneous if $\psi(\lambda r)=\lambda \psi(r)$ for all $\lambda \geq 0$. Since $\psi$ is always nonnegative in this paper, we simply say homogeneous to mean positively homogeneous. Define

$$
B_{\psi}:=\left\{x \in \mathbb{Q}^{2}: \psi(x-f) \leq 1\right\}
$$


The convexity of $\psi$ implies that $B_{\psi}$ is a convex set in $\mathbb{Q}^{2}$. The following result was proved in [4].

Theorem 1.1. Let $f \in \mathbb{Q}^{2}$. A minimal valid function $\psi$ for $R_{f}$ is nonnegative, homogeneous, piecewise linear, and convex. Furthermore, the closure of the set $B_{\psi}$ in $\mathbb{R}^{2}$ is a full-dimensional polyhedron with 2, 3 or 4 edges, it contains no integral point in its interior but each edge contains at least one integral point in its relative interior.

We will simply say in the interior of an edge to mean in its relative interior. Next we describe the different sets $B_{\psi}$ arising in Theorem 1.1.
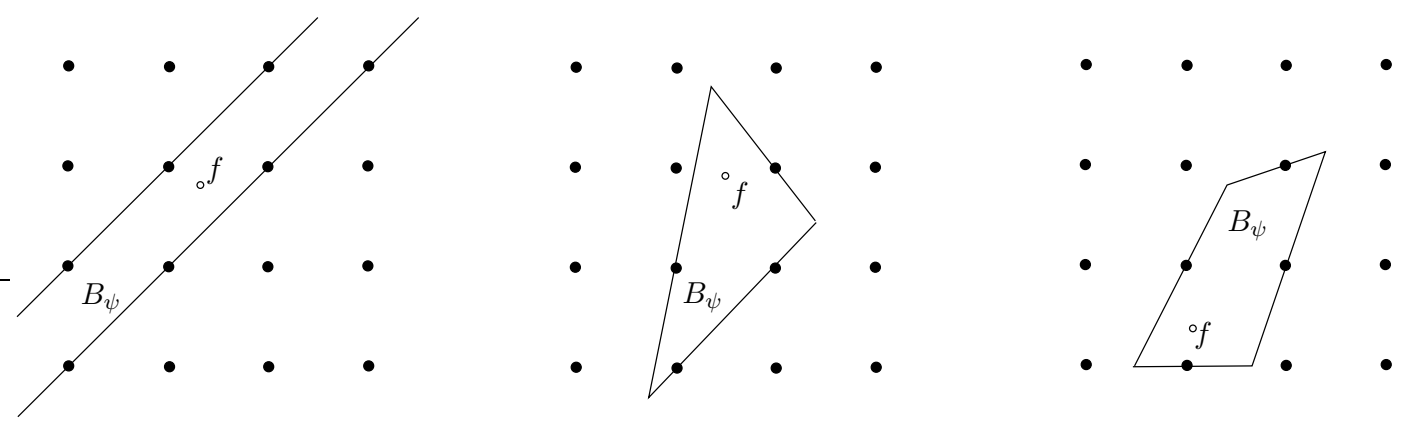

Figure 1: Representation of $B_{\psi}$ for nondegenerate cases.

The point $f$ is in $B_{\psi}$ since $\psi(0)=0$. The nondegenerate case is obtained when $f$ is in the interior of $B_{\psi}$ (see Figure 1). In this case, an extension of $\psi$ to $\mathbb{R}^{2}$ is obtained by defining $\psi(x-f)=1$ for all points on the boundary of the closure of $B_{\psi}$, denoted by $\operatorname{cl} B_{\psi}$. Indeed, the knowledge of $f$ and of the boundary of $\operatorname{cl} B_{\psi}$ together with the homogeneity of $\psi$ is enough to compute the value of $\psi(r)$ for any vector $r \in \mathbb{R}^{2} \backslash\{0\}$ : If there is a positive scalar $\lambda$ such that the point $f+\lambda r$ is on the boundary of $\operatorname{cl} B_{\psi}$, we get that $\psi(r)=1 / \lambda$. Otherwise, if there is no such $\lambda, r$ is an unbounded direction of $\operatorname{cl} B_{\psi}$ and $\psi(r)=0$. Note that this extension of $\psi$ to $\mathbb{R}^{2}$ is a continuous function.
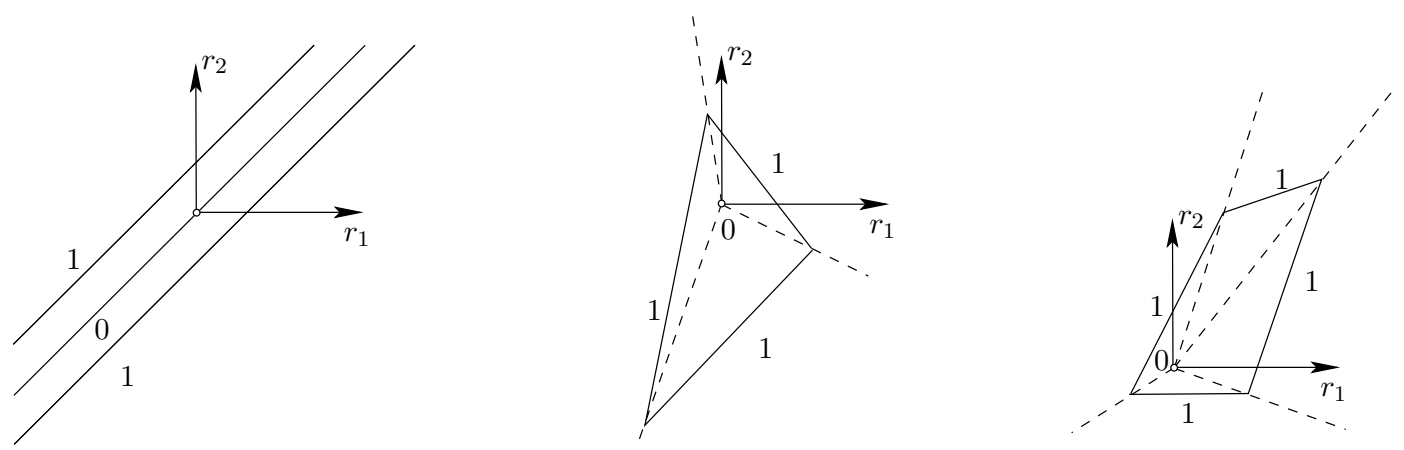

Figure 2: Level curves of $\psi(r)$ for nondegenerate cases.

We use the graphic representation of $B_{\psi}$ to describe $\psi$ when possible. The inequalities corresponding to the three cases of Figure 1 will be called split, triangle and quadrilateral 
inequalities. They are special cases of the intersection cuts of Balas [2]. Split inequalities in higher dimensions were studied by Cook, Kannan and Schrijver [6], who coined the name. These inequalities are equivalent to Gomory's mixed integer inequalities [10] (see [5] for a proof). In Figure 2, we represent the function $\psi$ in the $\left(r_{1}, r_{2}\right)$-space where $r=\left(\begin{array}{l}r_{1} \\ r_{2}\end{array}\right) \in \mathbb{R}^{2}$. Solid lines give level curves of $\psi(r)$ with values 0 and 1 for the three examples of Figure 1.

The degenerate case is obtained when $f$ is a vertex of $\operatorname{cl} B_{\psi}$ or when $f$ lies on one of its edges (see Figure 3 for three examples). Then $\psi$ is not finite everywhere [4]. An extension of $\psi$ to $\mathbb{R}^{2} \cup\{+\infty\}$ is obtained by defining $\psi(x-f)=1$ for all points on the boundary of $\mathrm{cl} B_{\psi}$, except for directions $(x-f)$ supporting edges of $\operatorname{cl} B_{\psi}$ containing $f$ : For any direction $0 \neq r \in \mathbb{R}^{2}$ such that the half-line $L_{r}=\{x=f+\lambda r$ for $\lambda>0\}$ is entirely outside $\operatorname{cl} B_{\psi}$, we have $\psi(r)=+\infty$. For the directions such that the half-line $L_{r}$ goes through the interior of $\operatorname{cl} B_{\psi}$, let $f+\lambda r$ be the point where $L_{r}$ intersects the boundary of $\operatorname{cl} B_{\psi}$; then we get $\psi(r)=1 / \lambda$. Finally, when $L_{r}$ supports an edge $E$ of $\operatorname{cl} B_{\psi}$, assume that $y=f+\lambda r$ is the first integral point encountered on $L_{r}$ starting from $f$ (such a point exists since $E$ contains an integral point and $f$ is rational, implying that $L_{r}$ contains infinitely many integral points) and let $x=f+\mu r$ be the first vertex of $\operatorname{cl} B_{\psi}$ encountered (if any); if $y$ is encountered first, we get $\psi(r)=1 / \lambda$ and if $x$ is encountered first, we get $\psi(r)=1 / \mu$. The resulting extension of $\psi$ to $\mathbb{R}^{2} \cup\{+\infty\}$ is not continuous, even in the region where $\psi$ is finite. Dey et al. [7] showed in a more general context that, if $\psi(r)<+\infty$ everywhere, then $\psi$ is continuous, and therefore $\psi$ is nondegenerate.

There are five different degenerate inequalities, depending on the type of set $\operatorname{cl} B_{\psi}$ and the position of $f$ on its faces: degenerate split, vertex-degenerate triangle, edge-degenerate triangle, vertex-degenerate quadrilateral and edge-degenerate quadrilateral inequalities. Solid lines in Figure 4 give level curves of $\psi(r)$ with value 1 for the three examples of Figure 3.
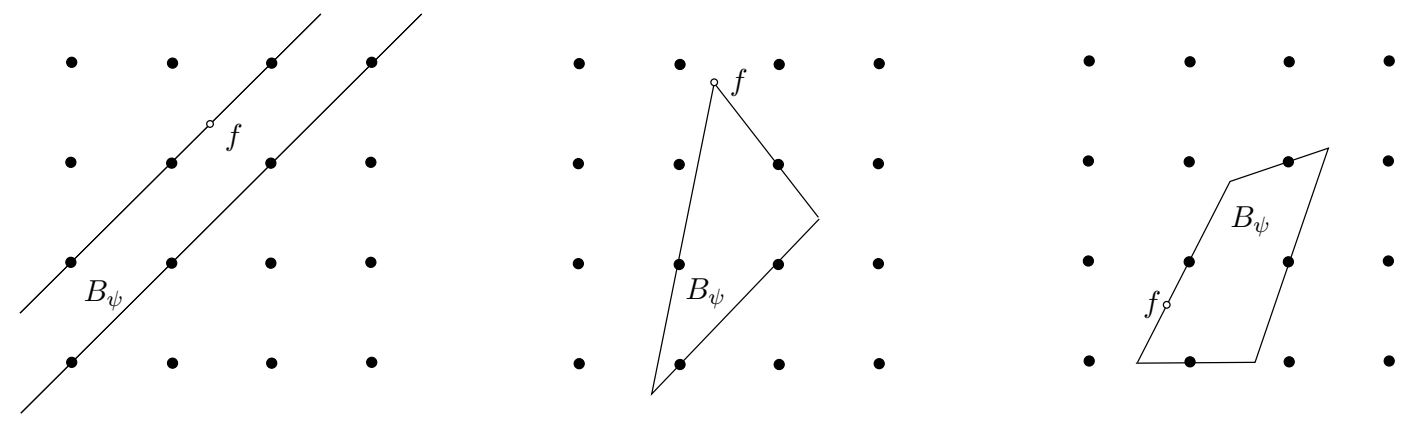

Figure 3: Representation of $B_{\psi}$ for degenerate cases.

A convex set with no integral point in its interior is called lattice-free. Maximal lattice-free convex sets in $\mathbb{R}^{n}$ are polyhedra with at most $2^{n}$ facets (Bell [3], Scarf [18] and Lovász [14]). The complete list of all maximal lattice-free convex sets in the plane is known:

Theorem 1.2. (Lovász [14]) A maximal lattice-free convex set in the $\left(x_{1}, x_{2}\right)$-plane $\mathbb{R}^{2}$ is one of the following:

i) A line $a x_{1}+b x_{2}=c$, where $a / b$ is irrational; 

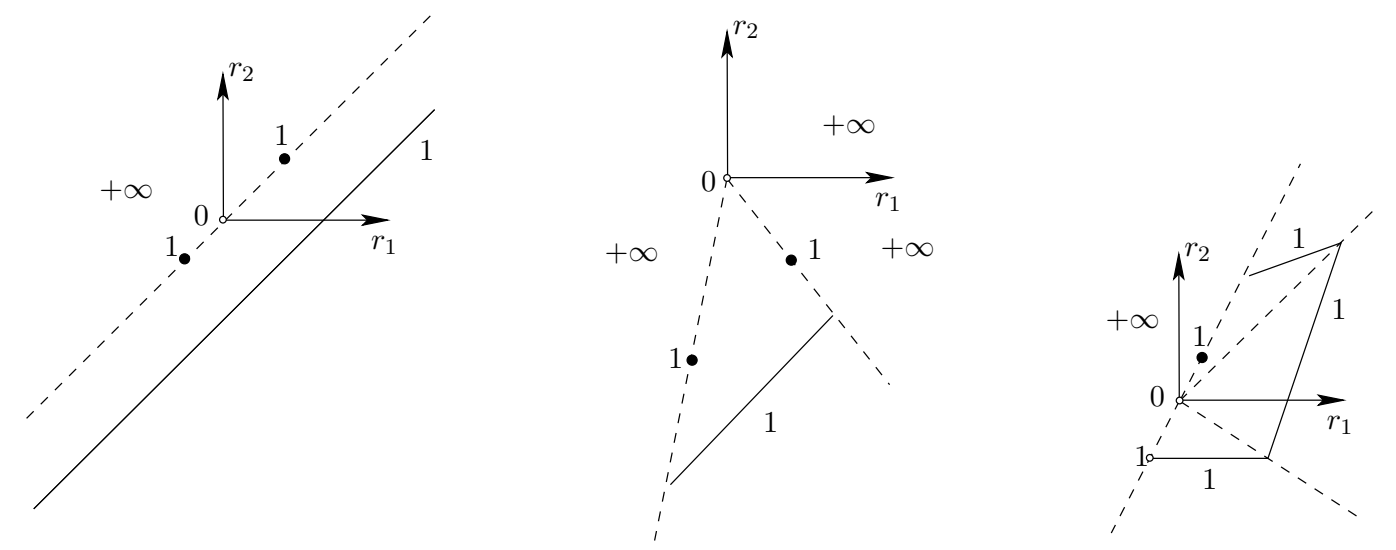

Figure 4: Level curves of $\psi(r)$ for degenerate cases.

ii) A strip $c \leq a x_{1}+b x_{2} \leq c+1$ where $a$ and $b$ are coprime integers and $c$ is an integer;

iii) A triangle with a least one integral point in the interior of each of its edges;

iv) A quadrilateral containing exactly four integral points, with exactly one of them in the interior of each of its edges; Moreover, these four integral points are vertices of a parallelogram of area 1 .

The polyhedra referred to in Theorem 1.1 correspond to the last three cases of Theorem 1.2. The first case does not play a role here as we only consider rational vectors $f$ and $r$ in the definition of $R_{f}$. Note that if we had defined $R_{f}$ and $R_{f}\left(r^{1}, \ldots, r^{k}\right)$ for parameters in $\mathbb{R}^{2}$ instead of $\mathbb{Q}^{2}$, the strict inequality $\sum_{r \neq r^{0}} s_{r}>0$ would be valid when $r_{1}^{0} / r_{2}^{0}$ is irrational and the line $f+\lambda r^{0}$ contains no integral point (this inequality corresponds to the first case of Theorem 1.2 and it is not implied by the valid inequalities of Theorem 1.1 since these inequalities all have $\psi\left(r^{0}\right)>0$ ). In particular $R_{f}\left(r^{1}, \ldots, r^{k}\right)$ would not be a polyhedron anymore. Thus the assumption that the vectors $f$ and $r$ are rational is important in the definitions of $R_{f}$ and $R_{f}\left(r^{1}, \ldots, r^{k}\right)$.

On the other hand, extending the definition of $\psi$ and $B_{\psi}$ to $\mathbb{R}^{2}$ is useful since the maximal lattice-free triangles and quadrilaterals in cases ii) and iii) of Theorem 1.2 may have irrational corner vertices. In the remainder of the paper, $\psi$ and $B_{\psi}$ are always assumed to be defined over $\mathbb{R}^{2}$.

\section{$1.2 \quad$ Facets}

A valid inequality $\sum \psi(r) s_{r} \geq 1$ for $R_{f}$ is extremal, or defines a facet of $R_{f}$ if there does not exist two distinct valid inequalities $\sum \psi_{j}(r) s_{r} \geq 1, j=1,2$, such that $\psi=\frac{1}{2} \psi_{1}+\frac{1}{2} \psi_{2}$. By extension, we also say that the function $\psi$ itself defines a facet of $R_{f}$. Note that, although we only use nontrivial inequalities in this definition, including them would give an equivalent definition.

Remark 1.3. The definition of a facet implies that if $\psi$ defines a facet of $R_{f}$ then $\psi$ is minimal. 
Remark 1.4. If a valid function $\psi$ is not facet defining for $R_{f}$, then $\psi \geq \frac{1}{2} \psi_{1}+\frac{1}{2} \psi_{2}$ where $\psi_{1}$ and $\psi_{2}$ are both minimal valid functions and $\psi \neq \psi_{1}$ or $\psi_{2}$.

Gomory [11] recently noted that triangle inequalities define facets of $R_{f}$ and raised the question of completely describing the facets of $R_{f}$. In this paper, we give such a characterization. It is summarized in the next theorem.

Theorem 1.5. The facets of $R_{f}$ are all split inequalities, all triangles inequalities, all quadrilateral inequalities that satisfy a certain ratio condition, all degenerate split inequalities, some degenerate triangle inequalities (see Theorems 4.9 and 4.10 for details) but no degenerate quadrilateral inequalities.

A valid inequality $\sum_{i=1}^{k} \psi\left(r^{i}\right) s_{i} \geq 1$ for $R_{f}\left(r^{1}, \ldots, r^{k}\right)$ defines a facet of $R_{f}\left(r^{1}, \ldots, r^{k}\right)$ if there does not exist two distinct valid inequalities $\sum_{i=1}^{k} \psi_{j}\left(r^{i}\right) s_{i} \geq 1, j=1,2$, such that $\psi\left(r^{i}\right)=\frac{1}{2} \psi_{1}\left(r^{i}\right)+\frac{1}{2} \psi_{2}\left(r^{i}\right)$ for $i=1, \ldots, k$. This definition of a facet of $R_{f}\left(r^{1}, \ldots, r^{k}\right)$ is consistent with the usual definition of a facet of a polyhedron only if the polyhedron is full dimensional. The next lemma shows that this is the case.

Lemma 1.6. If $R_{f}\left(r^{1}, \ldots, r^{k}\right)$ is non empty, then it is full dimensional.

Proof. The recession cone of $R_{f}\left(r^{1}, \ldots, r^{k}\right)$ is $\mathbb{R}_{+}^{k}$.

In the remainder, we assume that $R_{f}\left(r^{1}, \ldots, r^{k}\right) \neq \emptyset$. Note that this is easy to check. In particular, this is always the case when the rays $r^{j}$ for $j=1, \ldots, k$ span $\mathbb{R}^{2}$.

In [1], Andersen, Louveaux, Weismantel and Wolsey study $R_{f}\left(r^{1}, \ldots, r^{k}\right)$ and they prove that, when nonnegative combinations of $r^{1}, \ldots, r^{k}$ span $\mathbb{R}^{2}$, all the nontrivial facets of $R_{f}\left(r^{1}, \ldots, r^{k}\right)$ are split inequalities or are triangle or quadrilateral inequalities where the vertices of $B_{\psi}$ are on the rays $f+\lambda r^{i}, \lambda>0$, for $i=1, \ldots, k$. They do not, however, describe precisely which triangles and quadrilaterals generate facets. Some of the polyhedra $B_{\psi}$ they use are maximal lattice-free but, in order to have vertices of $B_{\psi}$ on rays $f+\lambda r^{i}, \lambda>0$, they also use polyhedra $B_{\psi}$ that are not maximal lattice-free. See Figure 5.
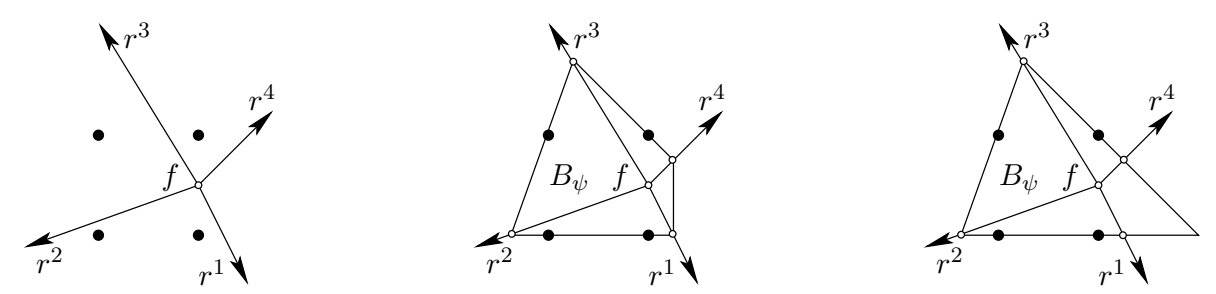

Figure 5: Data, a non maximal lattice-free quadrilateral defining a facet, and a maximal lattice-free triangle defining the same facet.

In addition to Theorem 1.5, the other main result of this paper is a characterization of the facets of $R_{f}\left(r^{1}, \ldots, r^{k}\right)$. 
Theorem 1.7. The facets of $R_{f}\left(r^{1}, \ldots, r^{k}\right)$ are

i) split inequalities where the infinite direction of $B_{\psi}$ is $r^{j}$ for some $j=1, \ldots, k$ and the line $f+\lambda r^{j}$ contains no integral point; or where $B_{\psi}$ satisfies a certain ray condition (see Theorem 3.12 for details)

ii) triangle inequalities where the triangle $B_{\psi}$ has its corner points on three half-lines $f+\lambda r^{j}$ for some $j=1, \ldots, k$ and $\lambda>0$; or where the triangle $B_{\psi}$ satisfies a certain ray condition (see Section 3.2.1 for details),

iii) quadrilateral inequalities where the corners of $B_{\psi}$ are on four half-lines $f+\lambda r^{j}$ for some $j=1, \ldots, k$ and $\lambda>0$, and $B_{\psi}$ satisfies a certain ratio condition (see Theorem 3.10 for details).

iv) None of the degenerate cases are needed to define the facets of $R_{f}\left(r^{1}, \ldots, r^{k}\right)$.

The paper is organized as follows. In Section 2, we give simple technical results that are useful in the remainder of the paper. In Section 3 we prove Theorem 1.7 and in Section 4 we prove Theorem 1.5.

\section{Preliminaries}

The three lemmas in this section collect simple properties that will be used in the remainder of the paper.

The first lemma is a characterization of maximal lattice-free triangles that was observed by Dey and Wolsey [8].

Lemma 2.1. If $T$ is a maximal lattice-free triangle in $\mathbb{R}^{2}$, then

i) Thas exactly three integral points on its boundary, one in the interior of each edge, or

ii) T has exactly six integral points on its boundary, the three vertices and one in the middle of each edge, or

iii) $T$ has exactly one edge $E$ with at least two integral points in its interior. The vertex opposite edge $E$ is nonintegral.

Proof. Suppose $T$ has an edge $E_{1}$ with at least two integral points $y^{1}, y^{2}$ in its interior, and another edge $E_{2}$ with two integral points $y^{3}, y^{4}$ distinct from the point $x^{3}=E_{1} \cap E_{2}$. Say $x^{3}, y^{1}, y^{2}$ appear in this order in $E_{1}$ and $x^{3}, y^{3}, y^{4}$ appear in this order in $E_{2}$. Then the points $y^{2}+\left(y^{3}-y^{1}\right)$ and $y^{4}+\left(y^{1}-y^{3}\right)$ are integral points and at least one of them is in the triangle $x^{3} y^{2} y^{4}$ and thus in the interior of $T$, a contradiction. This shows that either iii) holds or every edge of $T$ has exactly one integral point in its interior.

In the latter case, either i) holds or $T$ has an integral corner point $x^{3}$. Let $E_{1}$ and $E_{2}$ be the edges of $T$ containing $x^{3}$ and let $y^{i}$ be the integral vertex in the interior of $E_{i}$ for $i=1,2$. Let $x^{i}$ be the symmetric of $x^{3}$ relative to $y^{i}$. Since $x^{i}$ is integral and therefore not interior to $E_{i}$ by our assumption, $T$ is contained in the triangle $x^{1} x^{2} x^{3}$. It is easy to verify that the triangle $x^{1} x^{2} x^{3}$ only contains six integral points, namely the $x^{i} \mathrm{~s}, y^{1}, y^{2}$ and the middle 
$y^{3}$ of $x^{1}$ and $x^{2}$ : Indeed, the only integral points in triangle $x^{3} y^{1} y^{2}$ are its corners and by translation and symmetry the same holds for $x^{1} y^{1} y^{3}, y^{1} y^{2} y^{3}$ and $x^{2} y^{2} y^{3}$. By maximality, $T$ is the triangle $x^{1} x^{2} x^{3}$. Therefore ii) holds.

Lemma 2.2. Let $f \in \mathbb{Q}^{2}$ and let $\psi$ be valid and minimal for $R_{f}$. Let $x^{1}, x^{2}, x^{3} \in \mathbb{R}^{2}$ be three distinct points such that $x^{2}=\mu x^{1}+(1-\mu) x^{3}$ for some $0<\mu<1$.

i) If $\psi\left(x^{1}-f\right) \leq 1$ and $\psi\left(x^{2}-f\right)=1$ then $\psi\left(x^{3}-f\right) \geq 1$;

ii) If $\psi\left(x^{1}-f\right) \leq 1, \psi\left(x^{2}-f\right)=1$, and $\psi\left(x^{3}-f\right) \leq 1$ then $\psi\left(x^{1}-f\right)=\psi\left(x^{3}-f\right)=1$;

iii) If $\psi\left(x^{1}-f\right)=\psi\left(x^{2}-f\right)=\psi\left(x^{3}-f\right)=1$ then $\psi(x-f)=1$ for all $x$ on the line segment $\mu^{\prime} x^{1}+\left(1-\mu^{\prime}\right) x^{3}$ for $0 \leq \mu^{\prime} \leq 1$.

Proof. i) By Theorem 1.1, $\psi$ is convex and thus $1=\psi\left(x^{2}-f\right) \leq \mu \psi\left(x^{1}-f\right)+(1-\mu) \psi\left(x^{3}-f\right) \leq$ $\mu+(1-\mu) \psi\left(x^{3}-f\right)$. 1.

ii) Using i), we have $\psi\left(x^{3}-f\right) \geq 1$ and thus $\psi\left(x^{3}-f\right)=1$. Symmetry implies $\psi\left(x^{1}-f\right)=$

iii) Without loss of generality, assume that $x=\mu^{\prime} x^{1}+\left(1-\mu^{\prime}\right) x^{3}=\mu^{\prime \prime} x^{2}+\left(1-\mu^{\prime \prime}\right) x^{3}$ for some $0 \leq \mu^{\prime \prime} \leq 1$. Convexity of $\psi$ then implies that $\psi(x-f) \leq \mu^{\prime \prime} \psi\left(x^{2}-f\right)+\left(1-\mu^{\prime \prime}\right) \psi\left(x^{3}-\right.$ $f)=1$. Applying ii) to the triplet $x^{1}, x^{2}, x$, we get $\psi(x-f)=1$.

Lemma 2.3. Let $f \in \mathbb{Q}^{2}$ and let $\psi$ be valid for $R_{f}$. Assume that $\psi \geq \frac{1}{2} \psi_{1}+\frac{1}{2} \psi_{2}$ where $\psi_{j}$ is valid and minimal for $j=1,2$.

i) If $\psi(y-f)=1$ for an integral point $y$ then $\psi_{j}(y-f)=1$ for $j=1,2$.

ii) Let $y^{1}$ and $y^{2}$ two distinct integral points. Let $E$ be a line segment with $y^{1}, y^{2} \in E$ and with $y^{1}$ in the interior of $E$. If $\psi(x-f)=1$ for all $x \in E$ then $\psi_{j}(x-f)=1$ for all $x \in E, j=1,2$.

iii) Let $x^{1}$ and $y^{1}$ be two distinct points, with $y^{1}$ integral. Let $E$ be a line segment with $x^{1}, y^{1} \in E$ and with $y^{1}$ in the interior of $E$. If $\psi(x-f)=1$ for all $x \in E$ and $\psi_{1}\left(x^{1}-f\right)=\psi_{2}\left(x^{1}-f\right)=1$ then $\psi_{j}(x-f)=1$ for all $x \in E, j=1,2$.

iv) Let $L$ be a line with direction $d \in \mathbb{Q}^{2}$ and containing integral points. If $\psi(x-f)=1$ for all $x \in L$, then $\psi_{j}(x-f)=1$ for all $x \in L, j=1,2$.

Proof. i) Since $y$ is an integral point and $\psi_{j}$ is valid, $\psi_{j}(y-f) \geq 1$. As $\psi(y-f)=1$ and $\psi \geq \frac{1}{2} \psi_{1}+\frac{1}{2} \psi_{2}$, we get $\psi_{j}(y-f)=1$ for $j=1,2$.

ii) Let $x \in E$ with $x$ outside the line segment $y^{1} y^{2}$ with $y^{1}$ between $x$ and $y^{2}$. Such an $x$ exists as $y^{1}$ is in the interior of $E$. We claim that $\psi_{j}(x-f)=1$ for $j=1,2$. If $x$ is integral, point i) proves it. Assume now that $x$ is not integral. Lemma 2.2 i) for $\psi_{j}$ and the triplet $y^{2}, y^{1}, x$ shows that $\psi_{j}(x-f) \geq 1$. As $\psi(x-f)=1$ and $\psi \geq \frac{1}{2} \psi_{1}+\frac{1}{2} \psi_{2}$, we get $\psi_{j}(x-f)=1$. This proves the claim.

Using Lemma 2.2 iii), we get that $\psi_{j}(z-f)=1$ for all $z$ on the line segment $x y^{2}$ for $j=1,2$.

If there exists a point $x$ outside the segment $y^{1} y^{2}$ with $y^{2}$ between $x$ and $y^{1}$, a similar reasoning can be used to get $\psi_{j}(z-f)=1$ for all $z$ on the line segment $x y^{1}$ for $j=1,2$. 
iii) Similar to ii) with $x^{1}$ replacing $y^{2}$.

iv) Let $\bar{x} \in L$. Observe that since $d \in \mathbb{Q}^{2}$, there exist infinitely many integral points on $L$. It it thus possible to find three distinct integral points $y^{1}, y^{2}, y^{3} \in L$ such that both $y^{2}$ and $\bar{x}$ are in the interior of the line segment $E=y^{1} y^{3}$. Since $\psi(x-f)=1$ for all $x \in E$, point ii) above implies that $\psi_{j}(x-f)=1$ for all $x \in E$ (and thus for $\bar{x}$ ), for $j=1,2$.

\section{Facets of $R_{f}\left(r^{1}, \ldots, r^{k}\right)$}

In this section we prove Theorem 1.7. We first show that degenerate cases can be ignored when dealing with $R_{f}\left(r^{1}, \ldots, r^{k}\right)$. We then characterize when triangle and quadrilateral inequalities define facets. Finally we give conditions for split inequalities to define facets of $R_{f}\left(r^{1}, \ldots, r^{k}\right)$.

\subsection{Degenerate cases are not needed for $R_{f}\left(r^{1}, \ldots, r^{k}\right)$}

Theorem 3.1. Let $f, r^{1}, \ldots, r^{k} \in \mathbb{Q}^{2}$ with $k \geq 1$. Every nontrivial facet of $R_{f}\left(r^{1}, \ldots, r^{k}\right)$ can be obtained from a nondegenerate minimal valid function $\psi$.

Proof. It suffices to show that any degenerate minimal valid function $\psi$ is identical to a nondegenerate minimal valid function $\psi^{\prime}$ in the $k$ directions $r^{1}, \ldots, r^{k}$. Without loss of generality, assume that $\psi\left(r^{i}\right)<+\infty$ if and only if $i \leq \ell$ for some $0 \leq \ell \leq k$. We consider the five possible degenerate cases for $\psi$.

i) $\psi$ defines a degenerate split inequality. Let $L_{0}$ be the infinite edge of $\operatorname{cl} B_{\psi}$ containing $f$ and let $y^{1}$ and $y^{2}$ be the first integral points on $L_{0}$ encountered starting from $f$ in each direction, respectively. Let $L_{1}$ be the other infinite edge of $\operatorname{cl} B_{\psi}$ and let $y^{3}$ and $y^{4}$ be two distinct integral points on $L_{1}$. For $i=1, \ldots, \ell$, if there exists $\mu_{i}>0$ such that $f+\mu_{i} r^{i}$ is a point on $L_{1}$, let $w^{i}$ be this point. Otherwise, define $w^{i}=f$. The convex hull of $\left\{w^{i} \mid i=1, \ldots, \ell\right\} \cup\left\{y^{1}, \ldots, y^{4}\right\}$ is a lattice-free quadrilateral $Q$ with no integral point in the interior of the edge $E$ containing $f$. Replacing $E$ by an edge parallel to $E$ arbitrarily close to $E$, enlarging slightly $Q$ gives a lattice-free quadrilateral $Q^{\prime}$ with $f$ in its interior. Let $\psi^{\prime}$ be the minimal valid function corresponding to $Q^{\prime}$. We have $\psi^{\prime}\left(r^{i}\right) \leq \psi\left(r^{i}\right)$ for $i=1, \ldots, k$, proving the result.

ii) $\psi$ defines a vertex-degenerate triangle or quadrilateral inequality. Let $E_{1}$ and $E_{2}$ be the two edges of $\operatorname{cl} B_{\psi}$ incident with $f$ and let $y^{1}$ and $y^{2}$ be the first integral points encountered on these edges starting from $f$. Let $x$ be a point arbitrarily close to $f$ with $\psi(x-f)=+\infty$ and such that the cone $K$ with vertex $x$ and generated by the vectors $x y^{1}$ and $x y^{2}$ contains all positive multiples of the rays $r^{1}, \ldots, r^{\ell}$ except, possibly, those in directions $f y^{1}$ or $f y^{2}$. If $\psi$ defines a triangle inequality, let $L_{1}$ be the line supported by the edge of $\operatorname{cl} B_{\psi}$ not containing $f$ and let $H$ be the half-space limited by $L_{1}$ and containing $x$. Otherwise, $\psi$ defines a quadrilateral inequality, and let $H$ be the cone formed by the two edges of the quadrilateral not containing $f$. The intersection of $K$ with $H$ is a lattice-free triangle $T$ or quadrilateral $Q$ with $f$ in its interior. Let $\psi^{\prime}$ be the minimal function corresponding to $T$ or $Q$. We have $\psi^{\prime}\left(r^{i}\right) \leq \psi\left(r^{i}\right)$ for $i=1, \ldots, k$, proving the result.

iii) $\psi$ defines an edge-degenerate triangle or quadrilateral inequality. Consider first the case where the edge $E_{1}$ containing $f$ also contains an integral point interior to $E_{1}$ on each side 
of $f$. This situation can only occur when $\operatorname{cl} B_{\psi}$ is a triangle by Theorem $1.2 \mathrm{iv)}$. Let $y^{1}$ and $y^{2}$ be the first integral points on $E_{1}$ encountered starting from $f$ in each direction, respectively. Let $x$ be a point arbitrarily close to $f$ with $\psi(x-f)=+\infty$ and such that the cone $K$ with vertex $x$ and generated by the vectors $x y^{1}$ and $x y^{2}$ contains all the positive multiples of the rays $r^{1}, \ldots, r^{\ell}$ except, possibly, those in directions $f y^{1}$ or $f y^{2}$. The intersection of $K$ with the cone formed by the two edges of $\operatorname{cl} B_{\psi}$ not containing $f$ is a lattice-free quadrilateral $Q$ with $f$ in the interior of $Q$. Let $\psi^{\prime}$ be the minimal valid function corresponding to $Q$. We have $\psi^{\prime}\left(r^{i}\right) \leq \psi\left(r^{i}\right)$ for $i=1, \ldots, k$, proving the result.

Finally consider the case where the interior of the edge $E_{1}$ that contains $f$ has all its integral points on one side of $f$. Let $y$ be the closest integral point of $f$ in the interior of $E_{1}$. By tilting the edge $E_{1}$ around $y$ by a small angle we modify the original triangle $T$ (resp., quadrilateral $Q$ ) into a new triangle $T^{\prime}$ (resp. quadrilateral $Q^{\prime}$ ) with $f$ in its interior. Let $L_{1}^{\prime}$ be the line containing this tilted edge. Choose the tilting angle small enough so that all rays $r^{1}, \ldots, r^{\ell}$ except, possibly, those in the direction $f y$ point in the half-space limited by $L_{1}^{\prime}$ and containing $T^{\prime}$ (resp., $Q^{\prime}$ ). Let $\psi^{\prime}$ be the minimal valid function corresponding to $T^{\prime}$ (resp., $Q^{\prime}$ ). We have $\psi^{\prime}\left(r^{i}\right) \leq \psi\left(r^{i}\right)$ for $i=1, \ldots, k$, proving the result.

\subsection{Triangle and quadrilateral inequalities for $R_{f}\left(r^{1}, \ldots, r^{k}\right)$}

Let us turn now to the nondegenerate cases, i.e. $f$ is in the interior of $B_{\psi}$. We distinguish two cases depending on whether $B_{\psi}$ is unbounded or a polytope. We deal with the polytopes in this section and with the unbounded case in the next section.

\subsubsection{Reducing the dimension $k$}

Let $f, r^{1}, \ldots, r^{k} \in \mathbb{Q}^{2}$ with $k \geq 1$. Let $B_{\psi}$ be a maximal lattice-free triangle or quadrilateral with $f$ in its interior. In this section we reduce the question of whether $\psi$ defines a facet of $R_{f}\left(r^{1}, \ldots, r^{k}\right)$ to a problem on at most four rays $r^{j}$.

For $j=1, \ldots, k$, let $p^{j}$ be the intersection of the half-line $f+\lambda r^{j}, \lambda \geq 0$, with the boundary of $B_{\psi}$. The point $p^{j}$ is called the boundary point for $r^{j}$. Let $P$ be a set of boundary points. We say that a point $p \in P$ is active if it can have a positive coefficient in a convex combination of points in $P$ generating an integral point. Note that $p \in P$ is active if and only if $p$ is integral or there exists $q \in P$ such that the segment $p q$ contains an integral point in its interior. We say that an active point $p \in P$ is uniquely active if it has a positive coefficient in exactly one convex combination of points in $P$ generating an integral point.

We apply the following Reduction Algorithm:

0.) Let $P=\left\{p^{1}, \ldots, p^{k}\right\}$.

1.) While there exists $p \in P$ such that $p$ is active and $p$ is a convex combination of other points in $P$, remove $p$ from $P$. At the end of this step, $P$ contains at most two active points on each edge of $B_{\psi}$ and all points of $P$ are distinct.

2.) While there exists a uniquely active $p \in P$, remove $p$ from $P$.

3.) If $P$ contains exactly two active points $p$ and $q$ (and possibly inactive points), remove both $p$ and $q$ from $P$. 
We say that the ray condition holds for a triangle or a quadrilateral if $P=\emptyset$ at termination of the Reduction Algorithm.

Lemma 3.2. At termination of the Reduction Algorithm, the set of active points in $P$ is either empty or consists of the corner points of $B_{\psi}$.

Proof. Let $Q$ be the set of active points in $P$ at termination of the Reduction Algorithm. Suppose the lemma does not hold.

Observation 1: Step 1 implies that $Q$ has at most two points on each edge.

Observation 2: Step 2 implies that every point of $Q$ is involved in at least two distinct convex combinations.

Observation 3: Let $E$ be an edge of $B_{\psi}$ with a unique integral interior point. By Observations 1 and $2, Q$ cannot contain a point interior to $E$.

Observation 4: Step 3 and Observation 2 imply that $|Q| \geq 3$.

Suppose $B_{\psi}$ is a triangle of Type i) or ii) of Lemma 2.1. Observation 3 and the fact that $Q$ misses at least one corner of $B_{\psi}$ implies that all the points of $Q$ are on the same edge. This contradicts Observations 1 and 4 .

Suppose $B_{\psi}$ is a triangle of Type iii) of Lemma 2.1. By Observations 3 and $4, Q$ has two points in the edge with multiple integral points and one in the corner of $B_{\psi}$ opposite that edge. This last point contradicts Observation 2 since $Q$ misses at least one corner of $B_{\psi}$.

Suppose $B_{\psi}$ is a quadrilateral. By Observation 3, the points of $Q$ are corner points of $B_{\psi}$. Since $Q$ misses at least one corner of $B_{\psi}$, there is a point $q \in Q$ such that only one of the two edges containing $q$ also contains another point of $Q$. But then $q$ contradicts Observation 2 .

Let $x^{1}, \ldots, x^{h}$ denote the corner points of $B_{\psi}$. We define the corner rays of $B_{\psi}$ to be the rays $r^{j}=x^{j}-f$ for $j=1, \ldots, h$.

Theorem 3.3. Let $f, r^{1}, \ldots, r^{k} \in \mathbb{Q}^{2}$ with $k \geq 1$. Let $B_{\psi}$ be a maximal lattice-free triangle or quadrilateral with $f$ in its interior. Let $h=3$ when $B_{\psi}$ is a triangle and $h=4$ when $B_{\psi}$ is a quadrilateral. Then $\psi$ defines a facet of $R_{f}\left(r^{1}, \ldots, r^{k}\right)$ if and only if either

i) the set $\left\{r^{1}, \ldots, r^{k}\right\}$ contains rays $r^{i_{1}}, \ldots, r^{i_{h}}$ that are positive multiples of the corner rays of $B_{\psi}$ and $\psi$ defines a facet of $R_{f}\left(r^{i_{1}}, \ldots, r^{i_{h}}\right)$, or

ii) the ray condition holds.

Proof. The function $\psi$ defines a face of $R_{f}\left(r^{1}, \ldots, r^{k}\right)$. We study its dimension. Let $S=$ $\left\{s^{1}, \ldots, s^{t}\right\}$ in $R_{f}\left(r^{1}, \ldots, r^{k}\right)$ be a maximum cardinality set of affinely independent points that satisfy the inequality $\sum_{j=1}^{k} \psi\left(r^{j}\right) s_{j} \geq 1$ as an equality.

Let $R$ be the $2 \times k$ matrix whose column $j$ is $r^{j}$ for $j=1, \ldots k$. Let $S$ be the $k \times t$ matrix whose column $i$ is $s^{i}$ for $i=1, \ldots t$. Let $D$ be the $k \times k$ diagonal matrix whose $(j, j)$ entry is $\psi\left(r^{j}\right)$ for $j=1, \ldots k$. Observe that $D$ is invertible and let

$$
\bar{R}=R \cdot D^{-1} \quad \text { and } \quad \bar{S}=D \cdot S .
$$

Let us denote column $j$ of $\bar{R}$ by $\bar{r}^{j}$ and column $i$ of $\bar{S}$ by $\bar{s}^{i}$. Observe that for $j=1, \ldots, k$ we have $\psi\left(\bar{r}^{j}\right)=1$ implying that the point $p^{j}=f+\bar{r}^{j}$ is on the boundary of $B_{\psi}$. As 
multiplying each component of all the vectors in $S$ by a positive number does not change its affine dimension, the column set of $\bar{S}$ has the same affine dimension as the column set of $S$.

Claim 1: For $i=1, \ldots, t$, the coordinates of the vector $\bar{s}^{i}$ are the coefficients of a convex combination of the points in $P$ that yields an integral point on the boundary of $B_{\psi}$.

Indeed, we have

$$
\sum_{j=1}^{k} \bar{s}_{j}^{i}=\sum_{j=1}^{k} \psi\left(r^{j}\right) s_{j}^{i}=1
$$

Moreover, $\bar{s}^{i} \geq 0$ and $f+\sum_{j=1}^{k} r^{j} s_{j}^{i} \in \mathbb{Z}^{2}$. Thus

$$
\sum_{j=1}^{k} p^{j} \bar{s}_{j}^{i}=\sum_{j=1}^{k}\left(f+\bar{r}^{j}\right) \bar{s}_{j}^{i}=f+\sum_{j=1}^{k} r^{j} s_{j}^{i} \in \mathbb{Z}^{2} .
$$

Therefore the coordinates of $\bar{s}^{i}$ are the coefficients of a convex combination of points in $P$ that yields an integral point. Since the only integral points in $B_{\psi}$ are on its boundary, the claim follows.

Claim 1 implies that the columns of $\bar{S}$ are affinely independent if and only if they are linearly independent.

Claim 2: Suppose that the Reduction Algorithm removes point $p^{k} \in P$ in Step 1. If $k=1$, then $\psi$ defines a facet of $R_{f}\left(r^{1}, \ldots, r^{k}\right)$. Otherwise, $\psi$ defines a face of dimension $w$ of $R_{f}\left(r^{1}, \ldots, r^{k}\right)$ if and only if $\psi$ defines a face of dimension $w-1$ of $R_{f}\left(r^{1}, \ldots, r^{k-1}\right)$.

The boundary point $p^{k}$ is a convex combination of other points in $P$, say $p^{k}=\sum_{j=1}^{k-1} \alpha_{j} p^{j}$. Define the $k$-vector $v$ by

$$
v_{j}=\left\{\begin{array}{ll}
\alpha_{j} & \text { for } j=1, \ldots, k-1 \\
-1 & \text { for } j=k
\end{array} .\right.
$$

As $p^{k}$ is active, there exists a nonzero entry in row $k$ of $\bar{S}$. We can assume without loss of generality that $\bar{s}_{k}^{t}>0$. The vector $\bar{s}^{t+1}=\bar{s}^{t}+\bar{s}_{k}^{t} v$ is in $R_{f}\left(r^{1}, \ldots, r^{k}\right)$ and satisfies the inequality $\sum_{j=1}^{k} \psi\left(r^{j}\right) s_{j} \geq 1$ as an equality. Therefore it belongs to the affine space generated by the columns of $\bar{S}$. The vectors $\bar{s}^{t}, \bar{s}^{t+1}$ are linearly independent. It is thus possible to find a set $\bar{S}^{\prime}$ of $t$ linearly independent vectors in $\left\{\bar{s}^{1}, \ldots, \bar{s}^{t+1}\right\}$ and containing $\bar{s}^{t}, \bar{s}^{t+1}$. Then replacing $\bar{s}^{i}$ by $\bar{s}^{i}+\bar{s}_{k}^{i} v$ for all $\bar{s}^{i} \in \bar{S}^{\prime}$ except $\bar{s}^{t}$ is just doing elementary column operations and does not change the rank, since $v=\frac{1}{\bar{s}_{k}^{t}}\left(\bar{s}^{t+1}-\bar{s}^{t}\right)$. As the resulting vectors have a zero entry in component $k$ except $\bar{s}_{k}^{t}>0$, by removing $\bar{s}^{t}$ from $\bar{S}^{\prime}$ and deleting row $k$ in $\bar{S}^{\prime}$, we reduce by one both the dimension of the whole space and the dimension of the affine space spanned by $\bar{S}$. The converse is proved analogously, adding one zero component to the columns in $\bar{S}$ and one new column in $\bar{S}$ corresponding to a convex combination where $p^{k}$ is active. The dimension of the space is increased by one as is the number of affinely independent points on the face. This proves the claim. 
Claim 3: Suppose that the Reduction Algorithm removes point $p^{k} \in P$ in Step 2. If $k=1$, then $\psi$ defines a facet of $R_{f}\left(r^{1}, \ldots, r^{k}\right)$. Otherwise, $\psi$ defines a face of dimension $w$ of $R_{f}\left(r^{1}, \ldots, r^{k}\right)$ if and only if $\psi$ defines a face of dimension $w-1$ of $R_{f}\left(r^{1}, \ldots, r^{k-1}\right)$.

Point $p^{k}$ is involved in a single convex combination $\bar{s}^{i}$. If $k=1, p^{k}$ is integral and the result clearly holds. Otherwise, removing $\bar{s}^{i}$ and component $k$ in $\bar{S}$, we reduce by one both the dimension of the whole space and the dimension of the affine space spanned by $\bar{S}$. The converse is proved as above. This proves the claim.

Claim 4: Suppose that $k=2$ and the Reduction Algorithm removes points $p^{1}, p^{2} \in P$ in Step 3. Then $\psi$ defines a facet of $R_{f}\left(r^{1}, r^{2}\right)$.

Points $p^{1}, p^{2}$ are involved in two distinct convex combinations $\bar{s}^{1}, \bar{s}^{2}$, and the result clearly holds.

Using Claims 2, 3 and 4 recursively, we get that $\psi$ defines a facet of $R_{f}\left(r^{1}, \ldots, r^{k}\right)$ if and only the ray condition holds or $\psi$ defines a facet of $R_{f}\left(r^{i_{1}}, \ldots, r_{k^{\prime}}\right)$ where $P^{\prime}=\left\{p^{i_{1}}, \ldots, p^{i_{k^{\prime}}}\right\}$ is the set of remaining boundary points at termination of the Reduction Algorithm.

Let $S^{\prime}$ be the $k^{\prime} \times t^{\prime}$ matrix obtained from $\bar{S}$ while applying the Reduction Algorithm. If some $p \in P^{\prime}$ is not active, then the row of $S^{\prime}$ corresponding to $p$ has only zero entries. This implies $t^{\prime}<k^{\prime}$, proving that $\psi$ does not define a facet of $R_{f}\left(r^{i_{1}}, \ldots, r^{i_{k^{\prime}}}\right)$. Therefore $\psi$ defines a facet of $R_{f}\left(r^{1}, \ldots, r^{k}\right)$ if and only if all points in $P^{\prime}$ are active and either the ray condition holds or $\psi$ defines a facet of $R_{f}\left(r^{i_{1}}, \ldots, r^{i_{k^{\prime}}}\right)$. Now the theorem follows from Lemma 3.2.

\subsubsection{Rationality of the triangles and quadrilaterals that define facets of $R_{f}\left(r^{1}, \ldots, r^{k}\right)$}

We say that a triangle or quadrilateral is rational if its boundary lines have rational equations, or equivalently, if the coordinates of its corner points are rational.

Lemma 3.4. Let $f, r^{1}, \ldots, r^{k} \in \mathbb{Q}^{2}$. The maximal lattice-free triangles and quadrilaterals of Theorems 3.3 i) are rational.

Proof. By a theorem of Meyer [16], the rationality of $f, r^{1}, \ldots, r^{k}$ implies that $R_{f}\left(r^{1}, \ldots, r^{k}\right)$ is a rational polyhedron. Therefore its facets $\sum_{j=1}^{k} \psi\left(r^{j}\right) s_{j} \geq 1$ have rational coefficients $\psi\left(r^{j}\right)$. For triangles and quadrilateral inequalities, these coefficients are strictly positive. Therefore $x^{j}=f+\frac{1}{\psi\left(r^{j}\right)} r^{j}$ has rational coordinates for $j=1, \ldots, k$. In particular, the coordinates of the corner points are rational.

As usual, let $f, r^{1}, \ldots, r^{k} \in \mathbb{Q}^{2}$. Suppose that $B_{\psi}$ is a maximal lattice-free triangle or quadrilateral with $f$ in its interior such that the ray condition holds. By Theorem 3.3, $\psi$ defines a facet of $R_{f}\left(r^{1}, \ldots, r^{k}\right)$. It may happen that $B_{\psi}$ is irrational, but there exists a maximal lattice-free rational triangle or quadrilateral $B_{\psi^{\prime}}$ defining the same facet of $R_{f}\left(r^{1}, \ldots, r^{k}\right)$ as $B_{\psi}$. The proof of this claim is straightforward, since the only edges of $B_{\psi}$ that can possibly be irrational have only one integral point $y$ in their interior and no ray intersects them except possibly at $y$. Pivoting the edge slightly around $y$, we can make the edge rational. 


\subsubsection{Corner rays}

In this section, we assume that $B_{\psi}$ is a maximal lattice-free triangle or quadrilateral and that $f$ is in its interior.

Let $x^{1}, \ldots, x^{h}$ be the vertices of $B_{\psi}$ and let $r^{1}, \ldots, r^{h}$ be the corner rays of $B_{\psi}$ with $r^{j}=x^{j}-f$. We always assume that the vertices are topologically ordered so that the edges of the boundary of $B_{\psi}$ are convex combinations of $x^{i}$ and $x^{i+1}$ with indices taken modulo $h$. Let $y^{i}$ be an integral point that can be obtained as a nontrivial convex combination of $x^{i}$ and $x^{i+1}$ for $i=1, \ldots, h$ (indices are always implicitly taken modulo $h$ ).

Define $Y$ as the $2 \times h$ matrix whose column $i$ is the vector $y^{i}$ for $i=1, \ldots, h$ (Recall that $h=3$ or 4 ). Define $X$ as the $2 \times h$ matrix whose column $i$ is the vector $x^{i}$ for $i=1, \ldots, h$. Let $S$ be the $h \times h$ matrix whose column $i$ is the vector corresponding to the coefficients in the convex combination of $x^{i}$ and $x^{i+1}$ giving $y^{i}$ for $i=1, \ldots, h$.

We then have

$$
Y=X \cdot S
$$

with

$$
S=\left(\begin{array}{ccc}
\alpha & 0 & 1-\gamma \\
1-\alpha & \beta & 0 \\
0 & 1-\beta & \gamma
\end{array}\right) \quad \text { or } \quad S=\left(\begin{array}{cccc}
\alpha & 0 & 0 & 1-\delta \\
1-\alpha & \beta & 0 & 0 \\
0 & 1-\beta & \gamma & 0 \\
0 & 0 & 1-\gamma & \delta
\end{array}\right)
$$

where $\alpha, \beta, \gamma$ and $\delta$ are all strictly between 0 and 1 .

Since we are interested in the dimension of faces of polyhedra, which requires checking affine independence of points, we add a third row full of 1 s to the matrices $Y$ (resp. $X$ ) to obtain matrix $\bar{Y}$ (resp., $\bar{X}$ ). Due to the specific form of the matrix $S$, we still have

$$
\bar{Y}=\bar{X} \cdot S \text {. }
$$

Let $A$ be an $m \times n$ matrix. The nullspace of $A$ is $\mathcal{N}(A)=\left\{x \in \mathbb{R}^{n} \mid A x=0\right\}$ and the columnspace of $A$ is $\mathcal{C}(A)=\left\{z \in \mathbb{R}^{m} \mid z=A x\right.$ for some $\left.x \in \mathbb{R}^{n}\right\}$.

The following three results are classical results of linear algebra [15]:

Lemma 3.5. Let $A$ be an $m \times n$ matrix and $B$ be an $n \times p$ matrix. Then

$$
\operatorname{rank}(A \cdot B)=\operatorname{rank}(B)-\operatorname{dim}(\mathcal{N}(A) \cap \mathcal{C}(B)) .
$$

Corollary 3.6. Let $A$ be an $m \times n$ matrix and $B$ an $n \times p$ matrix. If $\operatorname{rank}(A)=n$, then

$$
\operatorname{rank}(A \cdot B)=\operatorname{rank}(B) .
$$

Proof. If $\operatorname{rank}(A)=n$, then $\mathcal{N}(A)=\{0\}$ and has dimension 0. Applying Lemma 3.5 yields the result.

Corollary 3.7. Let $A$ be an $m \times n$ matrix and $B$ an $n \times p$ matrix. Then

$$
\operatorname{rank}(A \cdot B) \leq \min \{\operatorname{rank}(A), \operatorname{rank}(B)\} .
$$


Proof. Apply Lemma 3.5 to $A \cdot B$ and its transpose.

\section{Triangle inequalities}

Theorem 3.8. Triangle inequalities define facets of $R_{f}\left(r^{1}, r^{2}, r^{3}\right)$ when $r^{1}, r^{2}, r^{3}$ are the corner rays.

Proof. Since $h=3$ and $B_{\psi}$ is a triangle, both $\bar{Y}$ and $\bar{X}$ have rank 3. By Corollary 3.6, $S$ has rank 3 too. It implies that the columns of $S$ are affinely independent. Since they all satisfy with equality the inequality $\sum_{i=1}^{3} \psi\left(r^{i}\right) s_{i} \geq 1$, this inequality defines a facet of $R_{f}\left(r^{1}, r^{2}, r^{3}\right)$.

Putting together Theorems 3.3 and 3.8, we get:

Theorem 3.9. A triangle inequality defines a facet of $R_{f}\left(r^{1}, \ldots, r^{k}\right)$ if and only if one of the following holds:

i) the set $\left\{r^{1}, \ldots, r^{k}\right\}$ contains rays $r^{i_{1}}, \ldots, r^{i_{3}}$ that are positive multiples of the corner rays of the triangle;

ii) the ray condition holds.

\section{Quadrilateral inequalities}

When $k=4$, both $\bar{Y}$ and $\bar{X}$ have rank 3. By Lemma 3.5, we have

$$
3=\operatorname{rank}(\bar{Y})=\operatorname{rank}(\bar{X} \cdot S)=\operatorname{rank}(S)-\operatorname{dim}(\mathcal{N}(\bar{X}) \cap \mathcal{C}(S)) .
$$

Since $\operatorname{rank}(\bar{X})=3$, we have that $\mathcal{N}(\bar{X})$ is a one-dimensional linear space. Hence $\operatorname{dim}(\mathcal{N}(\bar{X}) \cap \mathcal{C}(S)) \leq 1$ and $\operatorname{rank}(S)=4$ if and only if $\mathcal{N}(\bar{X}) \subseteq \mathcal{C}(S)$

Theorem 3.10. Consider a maximal lattice-free quadrilateral with vertices $x^{i}$, integral point $y^{i}$ on edge $x^{i} x^{i+1}$ (indices taken modulo 4 ) and corner rays $r^{i}, i=1, \ldots, 4$. The corresponding quadrilateral inequality defines a facet of $R_{f}\left(r^{1}, r^{2}, r^{3}, r^{4}\right)$ if and only if there is no $t \in \mathbb{R}_{+}$ such that the point $y^{i}$ divides the edge joining $x^{i}$ to $x^{i+1}$ in a ratio $t$ for odd $i$ and in a ratio $1 / t$ for even $i$, i.e.

$$
\frac{\left\|y^{i}-x^{i}\right\|}{\left\|y^{i}-x^{i+1}\right\|}= \begin{cases}t & \text { for } i=1,3 \\ \frac{1}{t} & \text { for } i=2,4\end{cases}
$$

Proof. Let $F$ be the face of $R_{f}\left(r^{1}, \ldots, r^{4}\right)$ defined by $\sum_{i=1}^{4} \psi\left(r^{i}\right) s_{i}=1$. As $f+r^{i}=x^{i}$ is on the boundary of $B_{\psi}$, we have $\psi\left(r^{i}\right)=1$ for $i=1, \ldots, 4$. Hence, if $s \in F$ then $\sum_{i=1}^{4} s_{i}=1$. Recall that $R_{f}\left(r^{1}, \ldots, r^{k}\right)$ is the convex hull of vectors in the set $H:=\left\{s \in \mathbb{R}_{+}^{4} \mid f+\right.$ $\sum_{i=1}^{4} r^{i} s_{i}$ is integral $\}$. Thus, if $F$ is a facet, then there exist four affinely independent vectors $s^{j}$, for $j=1, \ldots, 4$, in $H$ with

$$
\sum_{i=1}^{4} s_{i}^{j}=1 \quad \text { and } \quad z^{j}=f+\sum_{i=1}^{4} r_{i} s_{i}^{j}=\sum_{i=1}^{4}\left(f+r_{i}\right) s_{i}^{j} \quad \text { integral } .
$$


This implies that $z^{j}$ is in the convex hull of $x^{1}, \ldots, x^{4}$, for $j=1, \ldots, 4$. Theorem 1.2 shows that the only integral points in $B_{\psi}$ are the points $y^{1}, \ldots, y^{4}$. Moreover, for each $j=1, \ldots, 4$, there is a unique convex combination of $x^{1}, \ldots, x^{4}$ that produces $y^{j}$, namely column $j$ of matrix $S$. In other words, $F$ is a facet if and only if the columns of $S$ are affinely independent. Observe that the columns of $S$ are affinely independent if and only if they are linearly independent since the sum of the entries in any column of $S$ is 1 . It follows that $F$ is a facet if and only if $\operatorname{rank}(S)=4$.

Let $u=(1,-1,1,-1)^{T}$. By Theorem $\left.1.2 \mathrm{iv}\right)$, the points $y^{1}, \ldots, y^{4}$ are the vertices of a parallelogram. This implies that $\bar{Y} \cdot u=0$. Then (6) gives $\bar{X} \cdot S \cdot u=0$. We now have two cases:

i) $S \cdot u=0$. Then $\operatorname{rank}(S) \leq 3$ and Corollary 3.7 shows that $\operatorname{rank}(S)=3$. Solving the linear system $S \cdot u=0$ gives $\alpha=1-\beta=\gamma=1-\delta$. This is equivalent to the ratio condition of the statement.

ii) $S \cdot u \neq 0$. Then for $v=S \cdot u$ we have $\bar{X} \cdot v=0$, and as $v \neq 0$, we have that $\mathcal{N}(\bar{X})$ is the linear space spanned by $v$. Since $v$ is obtained as a linear combination of the columns of $S$, we have $\mathcal{N}(\bar{X}) \subseteq \mathcal{C}(S)$ and by Lemma 3.5 we get $\operatorname{rank}(S)=4$. Since all the columns of $S$ satisfy with equality the inequality $\sum_{i=1}^{4} \psi\left(r^{i}\right) s_{i} \geq 1$, this inequality defines a facet of $R_{f}\left(r^{1}, r^{2}, r^{3}, r^{4}\right)$.

We say that a maximal lattice-free quadrilateral satisfies the ratio condition when (7) does not hold for any $t>0$.

We illustrate the ratio condition by a couple of examples. The quadrilateral inequality generated from the square whose edges contain the integral points $\left(\begin{array}{l}0 \\ 0\end{array}\right),\left(\begin{array}{l}1 \\ 0\end{array}\right),\left(\begin{array}{l}0 \\ 1\end{array}\right),\left(\begin{array}{l}1 \\ 1\end{array}\right)$ in their middle does not define a facet of $R_{f}$, independently of the position of $f$ in its interior. As mentioned in [4], when $f$ is in the center of the square, the resulting quadrilateral inequality is a convex combination of the two split inequalities whose respective unbounded directions are the two coordinate axes. However, if one tilts just one edge of the square around its (integral) middle point, the resulting trapezoid has three distinct ratios $\frac{\left\|y^{i}-x^{i}\right\|}{\left\|y^{i}-x^{i+1}\right\|}$. Therefore the ratio condition is satisfied and Theorem 3.10 states that the resulting quadrilateral inequality defines a facet of $R_{f}$.

We give another more complicated example, see Figure 6. Let $f=\left(\begin{array}{c}\frac{1}{2} \\ \frac{1}{2}\end{array}\right)$ and $Q$ the quadrilateral with vertices $x^{1}=\left(\begin{array}{c}\frac{7}{6} \\ \frac{1}{6}\end{array}\right), x^{2}=\left(\begin{array}{c}\frac{7}{8} \\ \frac{13}{8}\end{array}\right), x^{3}=\left(\begin{array}{r}-\frac{7}{6} \\ \frac{1}{6}\end{array}\right), x^{4}=\left(\begin{array}{r}\frac{7}{8} \\ -\frac{1}{8}\end{array}\right)$.

Edge $x^{1} x^{2}$ contains integral point $y^{1}=\left(\begin{array}{l}1 \\ 1\end{array}\right)$ with ratio $\frac{\left\|y^{1}-x^{1}\right\|}{\left\|y^{1}-x^{2}\right\|}=\frac{4}{3}$.

Edge $x^{2} x^{3}$ contains integral point $y^{2}=\left(\begin{array}{l}0 \\ 1\end{array}\right)$ with ratio $\frac{\left\|y^{2}-x^{2}\right\|}{\left\|y^{2}-x^{3}\right\|}=\frac{3}{4}$.

Edge $x^{3} x^{4}$ contains integral point $y^{3}=\left(\begin{array}{l}0 \\ 0\end{array}\right)$ with ratio $\frac{\left\|y^{3}-x^{3}\right\|}{\left\|y^{3}-x^{4}\right\|}=\frac{4}{3}$.

Edge $x^{4} x^{1}$ contains integral point $y^{4}=\left(\begin{array}{l}1 \\ 0\end{array}\right)$ with ratio $\frac{\left\|y^{4}-x^{4}\right\|}{\left\|y^{4}-x^{1}\right\|}=\frac{3}{4}$.

Theorem 3.10 states that the quadrilateral inequality obtained from $Q$ is not a facet. 


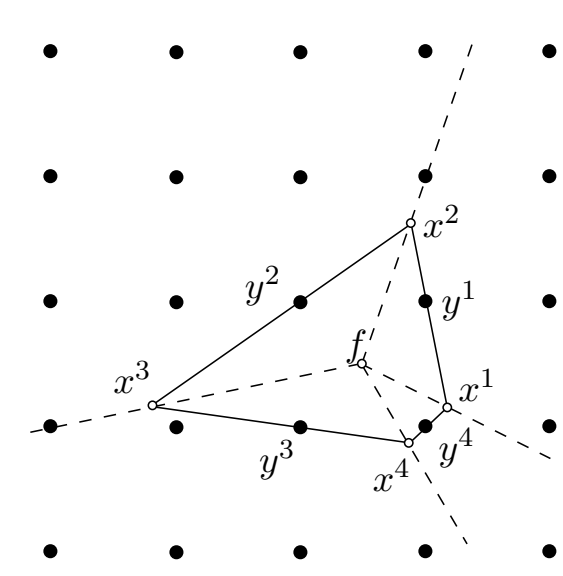

(a) The quadrilateral $Q$.

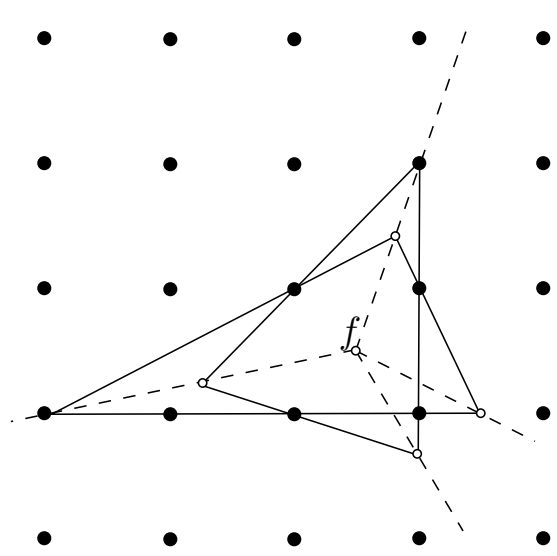

(b) The two triangles $T_{1}$ and $T_{2}$.

Figure 6: Illustration for the second example.

Indeed, it can be obtained as a convex combination of two triangle inequalities, each with a multiplier $\frac{1}{2}$. The first triangle $T_{1}$ has vertices $\left(\begin{array}{c}\frac{3}{2} \\ 0\end{array}\right),\left(\begin{array}{c}\frac{4}{5} \\ \frac{7}{5}\end{array}\right),\left(\begin{array}{r}-2 \\ 0\end{array}\right)$. The second triangle has vertices $\left(\begin{array}{r}1 \\ -\frac{1}{3}\end{array}\right),\left(\begin{array}{l}1 \\ 2\end{array}\right),\left(\begin{array}{r}-\frac{3}{4} \\ \frac{1}{4}\end{array}\right)$. Both triangles have all four points $y^{1}, y^{2}, y^{3}, y^{4}$ on their boundaries. The corner rays of $Q$ are $r^{1}=\left(\begin{array}{r}\frac{2}{3} \\ -\frac{1}{3}\end{array}\right), r^{2}=\left(\begin{array}{c}\frac{3}{8} \\ \frac{9}{8}\end{array}\right), r^{3}=\left(\begin{array}{c}-\frac{5}{3} \\ -\frac{1}{3}\end{array}\right)$, $r^{4}=\left(\begin{array}{r}\frac{3}{8} \\ -\frac{5}{8}\end{array}\right)$. Triangle $T_{1}$ has corner rays positive multiples of $r^{1}, r^{2}$ and $r^{3}$. Triangle $T_{2}$ has corner rays positive multiples of $r^{2}, r^{3}$ and $r^{4}$. If $\psi, \psi_{1}$ and $\psi_{2}$ denote the functions defined by $Q, T_{1}$ and $T_{2}$ respectively, it is easy to verify that $\psi=\frac{1}{2} \psi_{1}+\frac{1}{2} \psi_{2}$ in each of the cones $r^{i} r^{i+1}$ (indices defined modulo 4). Indeed, each of these functions is linear in each of the cones. So it is sufficient to verify the equality $\psi(r)=\frac{1}{2} \psi_{1}(r)+\frac{1}{2} \psi_{2}(r)$ in each of the directions $r^{i}, i=1, \ldots, 4$. In direction $r^{1}$ we have $\psi_{1}\left(\begin{array}{r}1 \\ -\frac{1}{2}\end{array}\right)=1$ and $\psi_{2}\left(\begin{array}{r}\frac{1}{2} \\ -\frac{1}{4}\end{array}\right)=1$. This implies $\psi_{1}\left(r^{1}\right)=\frac{2}{3}$ and $\psi_{2}\left(r^{1}\right)=\frac{4}{3}$. Therefore $\psi\left(r^{1}\right)=\frac{1}{2} \psi_{1}\left(r^{1}\right)+\frac{1}{2} \psi_{2}\left(r^{1}\right)$ as required. Similarly, for the other rays, we find $\psi_{1}\left(r^{2}\right)=\frac{5}{4}$ and $\psi_{2}\left(r^{2}\right)=\frac{3}{4} ; \psi_{1}\left(r^{3}\right)=\frac{4}{3}$ and $\psi_{2}\left(r^{3}\right)=\frac{2}{3}$; $\psi_{1}\left(r^{4}\right)=\frac{3}{4}$ and $\psi_{2}\left(r^{4}\right)=\frac{5}{4}$.

Putting together Theorems 3.3 and 3.10 , we get:

Theorem 3.11. A quadrilateral inequality defines a facet of $R_{f}\left(r^{1}, \ldots, r^{k}\right)$ if and only if one of the following holds:

i) the set $\left\{r^{1}, \ldots, r^{k}\right\}$ contains rays $r^{i_{1}}, \ldots, r^{i_{4}}$ that are positive multiples of the corner rays of the quadrilateral, and the ratio condition is satisfied;

ii) the ray condition holds. 
When the ray condition holds for a quadrilateral $B_{\psi}$, the half-lines $f+\lambda r^{j}, \lambda \geq 0$, can only intersect the boundary of $B_{\psi}$ at integral points. When this happens, it is easy to find a maximal lattice-free triangle producing the same facet. Therefore we do not need to mention the ray condition in iii) of Theorem 1.7.

\subsection{Split inequalities for $R_{f}\left(r^{1}, \ldots, r^{k}\right)$}

When $B_{\psi}$ is unbounded, $\psi$ defines a split inequality for $R_{f}$ by Theorem 1.1. We will see in Theorem 4.1 that split inequalities always define facets of $R_{f}$. The situation for $R_{f}\left(r^{1}, \ldots, r^{k}\right)$ is a little bit more complicated, as the next theorem shows.

When $\psi\left(r^{i}\right)>0$ for $i=1, \ldots, k$, we can define the boundary point for $r^{i}$ similarly to the case of triangles and quadrilaterals, and we can apply the Reduction Algorithm of Section 3.2.1. We say that the ray condition holds for a split if, at termination of the Reduction Algorithm, either $P=\emptyset$, or $P=\left\{p_{1}, q_{1}, p_{2}, q_{2}\right\}$ with $p_{1}, q_{1}$ on one of the boundary lines and $p_{2}, q_{2}$ on the other and both line segments $p_{1} q_{1}$ and $p_{2} q_{2}$ contain at least two integral points.

Theorem 3.12. Let $f, r^{1}, \ldots, r^{k} \in \mathbb{Q}^{2}$ with $k \geq 1$. Let $\psi$ define a split inequality. The inequality $\sum_{i=1}^{k} \psi\left(r^{i}\right) s_{i} \geq 1$ defines a facet of $R_{f}\left(r^{1}, \ldots, r^{k}\right)$ if and only if

i) $\psi\left(r^{i}\right)=0$ for some $i=1, \ldots, k$; or

ii) $\psi\left(r^{i}\right)>0$ for all $i=1, \ldots, k$ and the ray condition holds.

Proof. Suppose that $\psi\left(r^{i}\right)=0$ for some $i \in\{1, \ldots, k\}$. By Theorem 1.1, the only minimal valid inequality having the coefficient of $s_{i}$ equal to 0 is the split inequality defined by $\psi$. Therefore $\psi \geq \frac{1}{2} \psi_{1}+\frac{1}{2} \psi_{2}$ where $\psi_{1}$ and $\psi_{2}$ are both valid and minimal implies that $\psi=\psi_{1}=$ $\psi_{2}$. Remark 1.4 proves that $\psi$ is a facet of $R_{f}\left(r^{1}, \ldots, r^{k}\right)$.

Suppose now that $\psi\left(r^{i}\right)>0$ for $i=1, \ldots, k$. Using arguments similar to those used in the proof of Lemma 3.2, we obtain that at termination of the Reduction Algorithm, the set $Q$ of active points is either empty, or it contains exactly two points $p_{1}, q_{1}$ in $L_{1}$ and two points $p_{2}, q_{2}$ in $L_{2}$ where $L_{1}, L_{2}$ are the boundary lines of $B_{\psi}$, and both line segments $p_{1} q_{1}$ and $p_{2} q_{2}$ contain at least two integral points. Using arguments similar to those used in the proof of Theorem 3.3, we obtain that $\psi$ defines a facet if and only if, at termination of the Reduction Algorithm, the set $P$ is empty or only contains active points. In other words, $\psi$ defines a facet if and only if the ray condition holds.

\section{Facets of $R_{f}$}

\subsection{Split inequalities define facets of $R_{f}$}

\subsubsection{Nondegenerate case}

Let $f \in \mathbb{Q}^{2}$. Consider a direction $r^{0} \in \mathbb{Q}^{2} \backslash\{0\}$ such that the line $L_{0}:=\left\{x=f+\alpha r^{0}, \alpha \in \mathbb{R}\right\}$ contains no integral point. Let $L_{1}$ and $L_{2}$ be parallel lines to $L_{0}$, each containing integral points, such that the set of points between $L_{1}$ and $L_{2}$ contains no integral point in its interior and contains $L_{0}$. (See Figure 7.) Define $\psi\left(r_{0}\right)=\psi\left(-r_{0}\right)=0, \psi(x-f)=1$ for any $x \in L_{1} \cup L_{2}$.

Theorem 4.1. Let $f \in \mathbb{Q}^{2}$. Split inequalities define facets of $R_{f}$. 


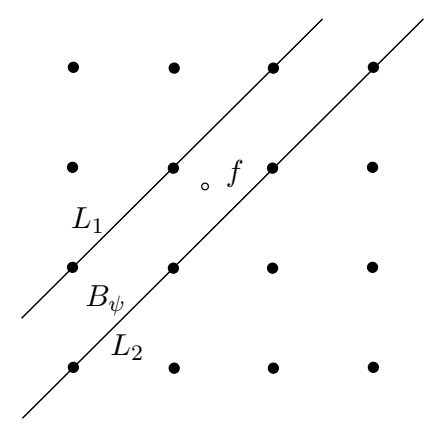

Figure 7: Illustration for Theorem 4.1.

Proof. Suppose not. By Remark 1.4, $\psi \geq \frac{1}{2} \psi_{1}+\frac{1}{2} \psi_{2}$ where $\psi_{1}, \psi_{2}$ are minimal valid functions and $\psi \neq \psi_{1}$ or $\psi_{2}$.

By Theorem 1.1, $\psi_{j}\left(r_{0}\right) \geq 0$ for $j=1,2$. Since $\psi\left(r_{0}\right)=0$, we have $\psi_{j}\left(r_{0}\right)=0$ for $j=1,2$. Similarly $\psi_{j}\left(-r_{0}\right)=0$.

Lemma $2.3 \mathrm{iv}$ ) for $L_{i}$ proves that $\psi_{j}(x-f)=1$ for all $x \in L_{i}$, for all $i=1,2, j=1,2$.

By homogeneity, $\psi_{j}$ is therefore defined over all of $\mathbb{R}^{2}$. But then $\psi_{1}=\psi_{2}=\psi$, a contradiction.

\subsubsection{Degenerate case}

Consider a direction $r^{0} \in \mathbb{Q}^{2} \backslash\{0\}$ such that the line $L_{0}:=\left\{x=f+\alpha r^{0}, \alpha \in \mathbb{R}\right\}$ contains integral points. Let $L_{1}$ be a line parallel to $L_{0}$ that contains integral points, such that the set of points between $L_{0}$ and $L_{1}$ contains no integral point in its interior. Let $y^{1}$ and $y^{2}$ be the first integral points encountered on the half-lines $f+\alpha r^{0}, \alpha \geq 0$, and $f-\alpha r^{0}, \alpha \geq 0$ respectively. (See Figure 8.) Define $\psi\left(y^{1}-f\right)=\psi\left(y^{2}-f\right)=1$ and $\psi(x-f)=1$ for any $x \in L_{1}$. Since $\psi$ is homogeneous, this defines $\psi(r)$ for all $r \in \mathbb{Q}^{2}$ in the closed half-space limited by $L_{0}$ and containing $L_{1}$. For all other $r \in \mathbb{Q}^{2} \backslash\{0\}$, define $\psi(r)=+\infty$. The inequality $\sum \psi(r) s_{r} \geq 1$, a degenerate split inequality, is valid for $R_{f}$.

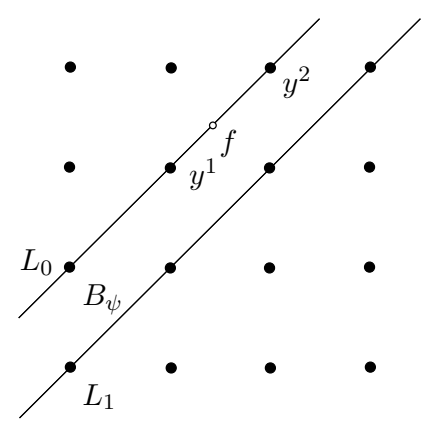

Figure 8: Illustration for Theorem 4.2.

Theorem 4.2. Let $f \in \mathbb{Q}^{2}$. Degenerate split inequalities define facets of $R_{f}$. 
Proof. Suppose not. Then $\psi \geq \frac{1}{2} \psi_{1}+\frac{1}{2} \psi_{2}$ where $\psi_{1}, \psi_{2}$ are minimal valid functions and $\psi \neq \psi_{1}$ or $\psi_{2}$.

Lemma 2.3 i) proves that $\psi_{j}\left(y^{1}-f\right)=1$ for $j=1,2$ and Lemma 2.3 iv) for $L_{1}$ proves that $\psi_{j}(x-f)=1$ for all $x \in L_{1}, j=1,2$.

By homogeneity, $\psi_{j}(x-f)$ is therefore defined for all $x \in \mathbb{R}^{2}$ that are in the closed halfspace $H$ limited by $L_{0}$ and containing $L_{1}$. Observe that $\psi(x-f)=\psi_{j}(x-f)$ for all $x \in \mathbb{R}^{2}$ and in $H$.

Suppose that there exists $x^{1} \notin H$ with $\psi_{j}\left(x^{1}-f\right)<+\infty$ for $j=1$ or $j=2$. Then $\psi_{j}(x-f) \leq \psi(x-f)$ for all $x \in \mathbb{R}^{2}$ with strict inequality for $x^{1}$, a contradiction to the minimality of $\psi$.

It follows that $\psi_{1}=\psi_{2}=\psi$, a contradiction.

\subsection{Nondegenerate triangle and quadrilateral inequalities}

Let $f \in \mathbb{Q}^{2}$. Nondegenerate minimal valid inequalities that are not split inequalities are generated by a function $\psi$ such that $B_{\psi}$ is either a triangle or a quadrilateral and $f$ is in the interior of $B_{\psi}$. Let $x^{1}, \ldots, x^{k}$ be the vertices of $B_{\psi}$ with $k=3$ or $k=4$. Note that these vertices may have irrational coordinates. Let $\left\{r^{1}, \ldots, r^{k}\right\}$ be the corner rays of $B_{\psi}$, namely $r^{i}=x^{i}-f$.

Given a valid and minimal function $\psi$ for $R_{f}$ and rays $r^{1}, \ldots, r^{k}$ we say that two valid and minimal functions $\psi_{1}$ and $\psi_{2}$ for $R_{f}$ dominate $\psi$ restricted to $\left\{r^{1}, \ldots, r^{k}\right\}$ if $\psi\left(r^{i}\right) \geq$ $\frac{1}{2} \psi_{1}\left(r^{i}\right)+\frac{1}{2} \psi_{2}\left(r^{i}\right)$ for $i=1, \ldots, k$ and $\psi\left(r^{i}\right)=\psi_{1}\left(r^{i}\right)$ and $\psi\left(r^{i}\right)=\psi_{2}\left(r^{i}\right)$ does not hold for all $i=1, \ldots, k$.

The next theorem will allow us to extend the proof of earlier results to cover the case of possibly irrational corner rays.

Theorem 4.3. Let $f \in \mathbb{Q}^{2}$ and let $\psi$ be a minimal valid function for $R_{f}$. Assume that $B_{\psi}$ is a polytope with $f$ in its interior and let $r^{1}, \ldots, r^{k}$ be the corner rays of $B_{\psi}$. Then there exist two minimal valid functions $\psi_{1}$ and $\psi_{2}$ dominating $\psi$ restricted to $\left\{r^{1}, \ldots, r^{k}\right\}$ if and only if $\psi$ is not facet defining for $R_{f}$.

Proof. Let $x^{1}, \ldots, x^{k}$ be the vertices of $B_{\psi}$. Let $E_{i}$ denote the edge of $B_{\psi}$ between vertices $x^{i}$ and $x^{i+1}$ for $i=1 \ldots, k$ and let $y^{i}$ be an integral point that can be obtained as a nontrivial convex combination of $x^{i}$ and $x^{i+1}$ for $i=1, \ldots, k$ (indices are implicitly taken modulo $k$ ).

Assume first that the two functions $\psi_{1}, \psi_{2}$ of the statement of the theorem exist. Let $\bar{\psi}=\frac{1}{2} \psi_{1}+\frac{1}{2} \psi_{2}$. Consider the edge joining $x^{i}$ to $x^{i+1}$. Since $\psi_{1}$ and $\psi_{2}$ are valid functions and $y^{i}$ is integral, we have $\psi_{1}\left(y^{i}-f\right) \geq 1$ and $\psi_{2}\left(y^{i}-f\right) \geq 1$. This implies $\bar{\psi}\left(y^{i}-f\right) \geq 1$. On the other hand, convexity of $\bar{\psi}$ implies $\bar{\psi}\left(y^{i}-f\right) \leq \lambda \bar{\psi}\left(x^{i}-f\right)+(1-\lambda) \bar{\psi}\left(x^{i+1}-f\right)$ where $0<\lambda<1$ is the convex combination of $x^{i}, x^{i+1}$ producing $y^{i}$. Since $\psi_{1}$ and $\psi_{2}$ dominate $\psi$ restricted to $\left\{r^{1}, \ldots, r^{k}\right\}$, we get $\bar{\psi}\left(y^{i}-f\right) \leq \lambda \psi\left(x^{i}-f\right)+(1-\lambda) \psi\left(x^{i+1}-f\right)=1$. Therefore $\bar{\psi}\left(y^{i}-f\right)=1$. Applying Lemma 2.2 i) to $\bar{\psi}$ and the triple $x^{i} y^{i} x^{i+1}$, we get $\bar{\psi}\left(r^{i+1}\right) \geq 1$. But as $1=\psi\left(r^{i+1}\right) \geq \bar{\psi}\left(r^{i+1}\right)$, we get $\bar{\psi}\left(r^{i+1}\right)=1$. Applying Lemma 2.2 iii) to $\psi$ and the triple $x^{i} y^{i} x^{i+1}$, we get that $\bar{\psi}(x-f)=\psi(x-f)$ for all $x$ in the segment $x^{i} x^{i+1}$. A similar reasoning shows that $\bar{\psi}(x-f)=1$ for all $x$ on the boundary of $B_{\psi}$, proving that $\bar{\psi}=\psi$. But then $\psi$ does not define a facet of $R_{f}$ as it is a convex combination of $\psi_{1}$ and $\psi_{2}$. 
For the converse, assume that $\psi$ does not define a facet of $R_{f}$ and let $\psi_{1} \neq \psi_{2}$ be two valid and minimal functions such that $\psi \geq \frac{1}{2} \psi_{1}+\frac{1}{2} \psi_{2}$ with $\psi_{1}(z-f) \neq 1$ for some $z \in B_{\psi}$. Then either $\psi_{1}(z-f)<1$ or $\psi_{2}(z-f)<1$. Assume without loss of generality that $\psi_{1}(z-f)<1$ and that $z \in E_{i}$. Applying Lemma 2.3 i) to $y^{i}$, we get $\psi_{1}\left(y^{i}-f\right)=1$. If we have $\psi_{1}\left(r^{i}\right)=\psi_{1}\left(r^{i+1}\right)=1$, applying Lemma 2.2 iii) to $\psi_{1}$ and the triple $x^{i} y^{i} x^{i+1}$, we get that $\psi_{1}(x-f)=1$ for all $x$ in the segment $x^{i} x^{i+1}$. This is a contradiction with $\psi_{1}(z-f)<1$. Hence, either $\psi_{1}\left(r^{i}\right) \neq \psi\left(r^{i}\right)=1$ or $\psi_{1}\left(r^{i+1}\right) \neq \psi\left(r^{i+1}\right)=1$ and $\psi_{1}, \psi_{2}$ are two valid minimal functions dominating $\psi$ restricted to $\left\{r^{1}, \ldots, r^{k}\right\}$.

The following is an extension of Theorem 3.8 to cover the case of triangles with possibly irrational corner rays.

Lemma 4.4. Let $\psi$ be valid and minimal such that $B_{\psi}$ is a triangle with corner rays $r^{1}, r^{2}, r^{3}$ and $f$ in its interior. Then $\psi$ restricted to $\left\{r^{1}, r^{2}, r^{3}\right\}$ cannot be dominated by two valid and minimal functions $\psi_{1}$ and $\psi_{2}$.

Proof. The proof of Theorem 3.8 can be used as is. The definitions of $\bar{Y}, \bar{X}$ and $S$ are not affected by the possible irrationality of some of the rays. It shows that the rank of $S$ is 3, i.e. the face $F$ of $\left\{s \in \mathbb{R}^{3} \mid f+\sum_{j=1}^{3} r^{j} s_{j} \in \mathbb{Z}^{2}, s \geq 0\right\}$ defined by $\sum \psi\left(r^{j}\right) s_{j} \geq 1$ has dimension 2. Its equation is uniquely defined. This implies that $\psi$ restricted to $\left\{r^{1}, r^{2}, r^{3}\right\}$ cannot be dominated by two valid and minimal functions $\psi_{1}, \psi_{2}$.

Similarly, the following is an extension of Theorem 3.10 to cover the case of quadrilaterals with possibly irrational corner rays.

Lemma 4.5. Let $\psi$ be valid and minimal such that $B_{\psi}$ is a quadrilateral with corner rays $r^{1}, r^{2}, r^{3}, r^{4}$ and $f$ in its interior. Then $\psi$ restricted to $\left\{r^{1}, r^{2}, r^{3}, r^{4}\right\}$ cannot be dominated by two valid and minimal functions $\psi_{1}$ and $\psi_{2}$ if and only if the quadrilateral satisfies the ratio condition.

Proof. The proof of Theorem 3.10 can be used as is. The definitions of $\bar{Y}, \bar{X}$ and $S$ are not affected by the possible irrationality of some of the rays. It shows that, depending on the ratio condition being satisfied or not, the rank of $S$ is 4 or 3, i.e. the face $F$ of $\left\{s \in \mathbb{R}^{4} \mid f+\sum_{j=1}^{4} r^{j} s_{j} \in \mathbb{Z}^{2}, s \geq 0\right\}$ defined by $\sum \psi\left(r^{j}\right) s_{j} \geq 1$ has dimension 3 or 2 .

If the ratio condition is satisfied, then a proof similar to the one for Lemma 4.4 shows that $\psi$ restricted to $\left\{r^{1}, r^{2}, r^{3}, r^{4}\right\}$ cannot be dominated by two valid and minimal functions.

If the ratio condition is not satisfied, there exists an hyperplane containing $F \cup\{0\}$ with equation $\sum_{j=1}^{4} h_{j} s_{j}=0$. For $\epsilon>0$, define $\psi_{1}, \psi_{2}$ by $\psi_{1}\left(r^{j}\right)=\psi\left(r^{j}\right)+\epsilon h_{j}$ and $\psi_{2}\left(r^{j}\right)=\psi\left(r^{j}\right)-\epsilon h_{j}$. As $\psi$ is valid, we have $\psi(y-f) \geq 1$ for all $y \in \mathbb{Z}^{2}$ and Theorem 1.2 iv) implies that equality holds only for the four integral points on the boundary of $B_{\psi}$, that is the four integral points obtained according to the convex combinations corresponding to the columns of $S$. This shows that for $\epsilon>0$ small enough, the two functions $\psi_{1}, \psi_{2}$ can be seen as restrictions of two valid quadrilateral inequalities. As $\psi\left(r^{j}\right)=\frac{1}{2} \psi_{1}\left(r^{j}\right)+\frac{1}{2} \psi_{2}\left(r^{j}\right)$ for $j=1, \ldots, 4$, it follows that $\psi$ restricted to $\left\{r^{1}, r^{2}, r^{3}, r^{4}\right\}$ is dominated by two valid and minimal functions.

As a consequence of the last three lemmas, we immediately get the following two results. 
Theorem 4.6. All maximal lattice-free triangles with $f$ in their interior define facets of $R_{f}$.

Theorem 4.7. A maximal lattice-free quadrilateral having $f$ in its interior defines a facet of $R_{f}$ if and only if it satisfies the ratio condition.

Although only rational triangles and quadrilaterals are needed to define the facets of $R_{f}\left(r^{1}, \ldots, r^{k}\right)$ when $f$ and the rays $r^{j}$ s are in $\mathbb{Q}^{2}$ (see Section 3.2.2), irrational triangles and quadrilaterals are needed to define some of the facets of $R_{f}$ for $f \in \mathbb{Q}^{2}$.

\subsection{Degenerate triangle inequalities}

In this section, we use the following notation:

Notation 4.8. Let $T$ be a maximal lattice-free triangle in $\mathbb{R}^{2}$. Let $x^{1}, x^{2}, x^{3}$ be the vertices of $T$ such that edge $E_{i}$ of $T$ is the convex combination of $x^{i}$ and $x^{i+1}$ for $i=1,2,3$ (indices are taken modulo 3). Let $y^{i}$ be an integral point in the interior of $E_{i}$ for $i=1,2,3$.

Note that the existence of $y^{i}$ for $i=1,2,3$ follows from Theorem 1.2.

\subsection{1 $f$ is a vertex of the triangle}

Here, we assume also that $f$ is a vertex of $T$. Without loss of generality, we assume $f=x^{2}$. Then edges $E_{1}$ and $E_{2}$ of $T$ contain $f$. (See Figure 9.)

Define $\psi\left(y^{1}-f\right)=\psi\left(y^{2}-f\right)=1$. By homogeneity, this defines $\psi(x-f)$ for all $x$ on the half-lines $L_{1}$ and $L_{2}$ starting from $f$ and containing $y^{1}$ and $y^{2}$ respectively. Define $\psi(x-f)=1$ for any $x$ in $E_{3} \backslash\left\{E_{1} \cup E_{2}\right\}$. By homogeneity, this defines $\psi$ in the open cone $C$ limited by $L_{1}$ and $L_{2}$. Finally, define $\psi(x-f)=+\infty$ for any $x$ outside $C \cup L_{1} \cup L_{2}$. Then $\psi$ defines a vertex-degenerate triangle inequality.

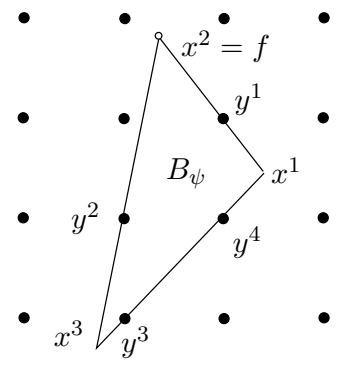

Figure 9: Illustration for Theorem 4.9.

Theorem 4.9. A vertex-degenerate triangle inequality defines a facet of $R_{f}$ if and only if the edge of $T$ opposite $f$ contains at least two integral points in its interior.

Proof. Suppose first that the edge $E_{3}$ contains only one integral point $y^{3}$ in its interior. Construct maximal lattice-free triangles $T^{\prime}$ and $T^{\prime \prime}$ from $T$ by keeping the half-lines $L_{1}$ and $L_{2}$ unchanged, and tilting the edge $E_{3}$ around the integral point $y^{3}$ so that the new edges $E_{1}^{\prime}$ ( $E_{1}^{\prime \prime}$ respectively) are slightly shorter (longer respectively) than $E_{1}$. The same construction used to define the function $\psi$ from $T$ can be used to define functions $\psi^{\prime}$ and $\psi^{\prime \prime}$ from $T^{\prime}$ and 
$T^{\prime \prime}$ respectively. The functions $\psi^{\prime}$ and $\psi^{\prime \prime}$ are linear in the open cone $C$ limited by $L_{1}$ and $L_{2}$ and $\psi\left(y^{3}\right)=\psi^{\prime}\left(y^{3}\right)=\psi^{\prime \prime}\left(y^{3}\right)=1$. Therefore, we can write $\psi$ as a convex combination of $\psi^{\prime}$ and $\psi^{\prime \prime}$. This shows that $\psi$ does not define a facet in this case.

Now consider the case where $E_{3}$ contains two integral points $y^{3}$ and $y^{4}$ in its interior. Suppose that $\psi$ does not define a facet of $R_{f}$. Then $\psi \geq \frac{1}{2} \psi_{1}+\frac{1}{2} \psi_{2}$ where $\psi_{j}$ is valid and minimal for $j=1,2$. Lemma 2.3 ii) shows that $\psi_{j}(x-f)=1$ for all $x$ in the interior of $E_{3}$.

By Lemma $2.3 \mathrm{i}$ ), $\psi_{j}\left(y^{1}-f\right)=1$, for $j=1,2$. By homogeneity, this defines $\psi_{j}(x-f)$ for all $x$ on the half-line $L_{1}$. Similarly $\psi_{j}\left(y^{2}-f\right)=1$ and by homogeneity, this defines $\psi_{j}(x-f)$ for all $x$ on the half-line $L_{2}$. Observe that $\psi(x-f)=\psi_{j}(x-f)$ for all $x \in \mathbb{R}^{2}$ and in $C \cup L_{1} \cup L_{2}$.

Suppose that there exists $x^{4} \notin C \cup L_{1} \cup L_{2}$ with $\psi_{j}\left(x^{4}-f\right)<+\infty$ for $j=1$ or $j=2$. Then $\psi_{j}(x-f) \leq \psi(x-f)$ for all $x \in \mathbb{R}^{2}$ with strict inequality for $x^{4}$, a contradiction with the minimality of $\psi$. It follows that $\psi_{1}=\psi_{2}=\psi$, a contradiction. Therefore $\psi$ define a facet of $R_{f}$.

\subsection{2 $f$ is on an edge of the triangle}

Here we use Notation 4.8 and we assume that $f$ is in the interior of an edge of $T$. Without loss of generality, we assume $f \in E_{1}$ and that $y^{1}$ is between $f$ and $x^{1}$. (See Figure 10.)

Define $\psi(x-f)=1$ for any $x \in\left(E_{2} \backslash\left\{x^{2}\right\}\right) \cup\left(E_{3} \backslash\left\{x^{1}\right\}\right)$. Let $L_{1}$ denote the line containing the segment $E_{1}$. Since $\psi$ is homogeneous, $\psi(x-f)$ is defined for all $x \in \mathbb{R}^{2}$ in the open half-space $H$ limited by $L_{1}$ and containing $x^{3}$.

Define $\psi\left(y^{1}-f\right)=1$. On the half-line from $f$ that goes through $x^{2}$, let $y^{4}$ be the first integral point encountered, starting from $f$. If $y^{4}$ is reached before $x^{2}$, then set $\psi\left(y^{4}-f\right)=1$. Otherwise set $\psi\left(x^{2}-f\right)=1$. By homogeneity, this defines $\psi(x-f)$ for all $x \in L_{1}$.

For all $x \in \mathbb{R}^{2} \backslash\left(L_{1} \cup H\right)$, define $\psi(x-f)=+\infty$. Then $\psi$ defines an edge-degenerate triangle inequality.

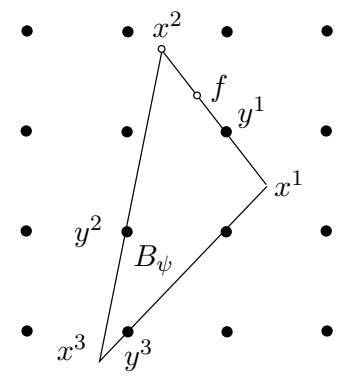

Figure 10: Illustration for Theorem 4.10.

Theorem 4.10. An edge-degenerate triangle inequality defines a facet of $R_{f}$ if and only if at least one of the two edges not containing $f$ contains at least two integral points $y$ with $\psi(y-f)=1$.

Proof. Suppose first that $E_{2}$ and $E_{3}$ each contain only one integral point, $y^{2}$ and $y^{3}$ respectively. Let $x^{4}$ and $x^{5}$ be points on the line $f x^{3}$ such that $x^{3}$ is their middle point and the 
distance between $x^{4}$ and $x^{5}$ is very small. Let $T^{\prime}$ ((resp. $\left.T^{\prime \prime}\right)$ denote the triangle with one side contained in $L_{1}$, one side containing $x^{4} y^{2}$ (resp. $x^{5} y^{2}$ ) and one side containing $x^{4} y^{3}$ (resp. $x^{5} y^{3}$ ). The convex sets $T^{\prime}$ and $T^{\prime \prime}$ define valid functions $\psi^{\prime}$ and $\psi^{\prime \prime}$ as above. Furthermore $\psi$ is a convex combination of $\psi^{\prime}$ and $\psi^{\prime \prime}$, showing that $\psi$ is not facet defining.

Suppose now that edge $E_{i}$ contains at least two integral points for $i=2$ or 3 . Without loss of generality, we can assume $i=2$. Suppose that $\psi$ does not define a facet of $R_{f}$. Then $\psi \geq \frac{1}{2} \psi_{1}+\frac{1}{2} \psi_{2}$ where $\psi_{j}$ are valid and minimal for $j=1,2$. Lemma 2.3 ii) shows that $\psi_{j}(x-f)=1$ for all $x \in E_{2} \backslash E_{1}$. In particular $\psi_{j}\left(x^{3}-f\right)=1$. for $j=1,2$.

The edge $E_{3}$ contains the integral point $y^{3}$ in its interior and the point $x^{3}$. Lemma 2.3 iii) shows that $\psi_{j}(x-f)=1$ for all $x \in E_{3} \backslash E_{1}$. This shows that $\psi_{1}(x-f)=\psi_{2}(x-f)=\psi(x-f)$ for all $x \in \mathbb{R}^{2}$ in the open half-space $H$ containing $x^{3}$ limited by $L_{1}$.

Lemma 2.3 i) shows that $\psi_{j}\left(y^{1}-f\right)=1$, for $j=1,2$. On the half-line of $L_{1}$ going through $x^{2}$ there are two cases. If an integral point $y^{4}$ is encountered starting from $f$ before reaching $x^{2}$, Lemma 2.3 i) yields $\psi_{j}\left(y^{4}-f\right)=1$, for $j=1,2$. So suppose that no integral point is encountered starting from $f$ before reaching $x^{2}$. In this case $\psi\left(x^{2}\right)=1$ and Lemma 2.3 iii) for $E_{2}$ and with $x^{3}$ playing the role of $x^{1}$ of the lemma shows that we have $\psi_{j}\left(x^{2}\right)=1$. By homogeneity, this defines $\psi_{j}(x-f)$ for all $x \in L_{1}$, for $j=1,2$.

Finally, the discontinuity of $\psi_{j}$ on the half-line of $L_{1}$ from $f$ in the direction of $y^{1}$ implies that $\psi_{j}(x-f)=+\infty$ for all $x \notin H \cup L_{1}$, using a similar argument as in the proof of Theorem 4.9.

\subsection{Degenerate quadrilateral inequalities}

In this section, we use the following notation:

Notation 4.11. Let $Q$ be a maximal lattice-free quadrilateral in $\mathbb{R}^{2}$. Let $x^{1}, \ldots, x^{4}$ be the vertices of $Q$ such that edge $E_{i}$ of $Q$ is the convex combination of $x^{i}$ and $x^{i+1}$ for $i=1, \ldots, 4$ (indices are taken modulo 4). Let $y^{i}$ be the unique integral point in the interior of $E_{i}$ for $i=1, \ldots, 4$.

Note that the existence and unicity of $y^{i}$ for $i=1, \ldots, 4$ follows from Theorem 1.2.

\subsection{1 $f$ is a vertex of the quadrilateral}

Here, we assume that $f$ is a vertex of $Q$. Without loss of generality, we assume $f=x^{2}$. (See Figure 11.)

Define $\psi\left(y^{1}-f\right)=\psi\left(y^{2}-f\right)=1$. By homogeneity, this defines $\psi(x-f)$ for all $x$ on the half-lines $L_{1}$ and $L_{2}$ starting from $f$ and containing $y^{1}$ and $y^{2}$ respectively. Define $\psi(x-f)=1$ for any $x$ in $E_{3} \cup E_{4} \backslash\left\{x^{1} \cup x^{3}\right\}$. By homogeneity, this defines $\psi$ in the open cone $C$ limited by $L_{1}$ and $L_{2}$. Finally, define $\psi(x-f)=+\infty$ for any $x$ outside $C \cup L_{1} \cup L_{2}$. Then $\psi$ defines a vertex-degenerate quadrilateral inequality.

Theorem 4.12. A vertex-degenerate quadrilateral inequality never defines a facet of $R_{f}$.

Proof. Let $x^{5}$ and $x^{6}$ be points on the line $f x^{4}$ such that $x^{4}$ is their middle point and the distance between $x^{5}$ and $x^{6}$ is very small. Let $Q^{\prime}$ denote the quadrilateral with vertices $x^{5}$, $f$ and two others on lines $f x^{1}$ and $f x^{2}$ respectively, and containing the integral points $y^{i}$ for 


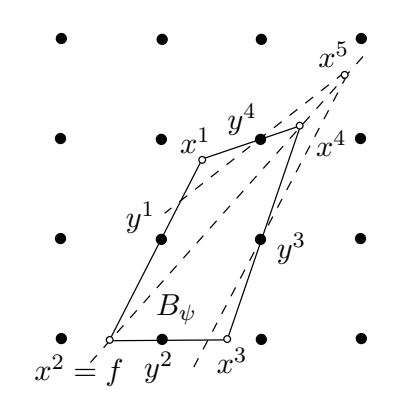

Figure 11: Illustration for Theorem 4.12 .

$i=1, \ldots, 4$ in the interior of its edges. Quadrilateral $Q^{\prime \prime}$ is defined similarly except that it has vertex $x^{6}$ instead of $x^{5}$. The convex sets $Q^{\prime}$ and $Q^{\prime \prime}$ define valid functions $\psi^{\prime}$ and $\psi^{\prime \prime}$ as above. Furthermore $\psi$ is a convex combination of $\psi^{\prime}$ and $\psi^{\prime \prime}$ with $\psi_{1} \neq \psi_{2}$, proving that the vertex-degenerate inequality is not a facet.

\subsection{2 $f$ is on an edge of the quadrilateral}

Here, we use Notation 4.11 and we assume that $f$ is on one of the edges of $Q$. Without loss of generality, we assume $f \in E_{1}$ and that $y^{1}$ is between $f$ and $x^{1}$.

Define $\psi\left(y^{1}-f\right)=1$. By homogeneity, this defines $\psi(x-f)$ for all $x$ on the half-line of $L_{1}$ starting from $f$ and containing $y^{1}$. Define $\psi(x-f)=1$ for any $x$ in $E_{2} \cup E_{3} \cup E_{4} \backslash\left\{x^{1}\right\}$. By homogeneity, this defines $\psi$ in the rest of the closed half-plane $H$ limited by the line $L_{1}$. Finally, define $\psi(x-f)=+\infty$ for any $x$ outside $C$. Then $\psi$ defines an edge-degenerate quadrilateral inequality.

Theorem 4.13. An edge-degenerate quadrilateral inequality never defines a facet of $R_{f}$.

Proof. As in the proof of Theorem 4.12, we can perturb $Q$ into two quadrilaterals $Q^{\prime}$ and $Q^{\prime \prime}$ going through $f$ and $y^{i}$ for $i=1, \ldots, 4$ and vertices on the lines $f x^{3}$ or $f x^{4}$ and construct the corresponding functions $\psi^{\prime}$ and $\psi^{\prime \prime}$ as above. Then $\psi$ is a convex combination of $\psi^{\prime}$ and $\psi^{\prime \prime}$ proving that the edge-degenerate inequality is not a facet.

Acknowledgements: We would like to thank Kent Andersen, Santanu Dey, Yanjun Li and Giacomo Zambelli for their helpful comments. In particular Kent Andersen's comments helped us bring into sharper focus the results for $R_{f}\left(r^{1}, \ldots, r^{k}\right)$ and Santanu Dey brought to our attention the importance of irrational polytopes for $R_{f}$.

\section{References}

[1] K. Andersen, Q. Louveaux, R. Weismantel and L. Wolsey, Cutting Planes from Two Rows of a Simplex Tableau, Proceedings of IPCO XII, Ithaca, New York (June 2007) $1-15$. 
[2] E. Balas, Intersection Cuts - A New Type of Cutting Planes for Integer Programming, Operations Research 19 (1971) 19-39.

[3] D.E. Bell, A Theorem Concerning the Integer Lattice, Studies in Applied Mathematics 56 (1977) 187-188.

[4] V. Borozan and G. Cornuéjols, Minimal Valid Inequalities for Integer Constraints, technical report (July 2007).

[5] G. Cornuéjols, Y. Li, On the Rank of Mixed 0,1 Polyhedra, Mathematical Programming A 91 (2002) 391-397.

[6] W. Cook, R. Kannan and A. Schrijver, Chvátal Closures for Mixed Integer Programming Problems, Mathematical Programming 47 (1990) 155-174.

[7] S.S. Dey and J.-P. P. Richard, L. A. Miller and Y. Li, Extreme Inequalities for Infinite Group Problems, technical report (2006).

[8] S.S. Dey and L.A. Wolsey, Lifting Integer Variables in Minimal Inequalities Corresponding to Lattice-Free Triangles, IPCO XIII, Bertinoro, Italy (June 2008).

[9] R.E. Gomory, Some Polyhedra Related to Combinatorial Problems, Journal of Linear Algebra and its Applications 2 (1969) 451-558.

[10] R.E. Gomory, An Algorithm for Integer Solutions to Linear Programs, Recent Advances in Mathematical Programming, R.L. Graves and P. Wolfe eds., McGraw-Hill, New York (1963) 269-302.

[11] R.E. Gomory, Thoughts about Integer Programming, 50th Anniversary Symposium of OR, University of Montreal, January 2007, and Corner Polyhedra and Two-Equation Cutting Planes, George Nemhauser Symposium, Atlanta, July 2007.

[12] R.E. Gomory and E.L. Johnson, Some Continuous Functions Related to Corner Polyhedra, Part I, Mathematical Programming 3 (1972) 23-85.

[13] E.L. Johnson, On the Group Problem for Mixed Integer Programming, Mathematical Programming Study (1974) 137-179.

[14] L. Lovász, Geometry of Numbers and Integer Programming, Mathematical Programming: Recent Developments and Applications, M. Iri and K. Tanabe eds., Kluwer (1989) 177210.

[15] C.D. Meyer, Matrix Analysis and Applied Linear Algebra, SIAM (2000).

[16] R.R. Meyer, On the Existence of Optimal Solutions to Integer and Mixed Integer Programming Problems, Mathematical Programming 7 (1974) 223-235.

[17] G.L. Nemhauser and L.A. Wolsey, Integer and Combinatorial Optimization, Wiley (1988).

[18] H.E. Scarf, An Observation on the Structure of Production Sets with Indivisibilities, Proc. Nat. Acad. Sciences USA 74 (1977) 3637-3641. 